\title{
The Transient Lubrication Problem as a Generalized Hele-Shaw Type Problem
}

\author{
G. Bayada, M. Boukrouche and M. El-A. Talibi
}

\begin{abstract}
The aim of this paper is to bridge the gap between Hele-Shaw theory and lubrication theory. A first generalization of the Hele-Shaw problem is considered for which existence, uniqueness and regularity theorems are given. Then taking shear effects into account, lubrication fluid mixture approach has to be used, inducing a new formulation of the initial problem. Connection of the two kind of problems are then given, establishing Hele-Shaw phenomena as a particular case of lubrication problem on a mathematical basis.
\end{abstract}

Keywords: Hele-Shaw flow, cavitation phenomena, free boundary Reynolds equations, Darcy.'s law, variational inequalities

AMS subject classiflcation: 35 Q 35, 35 R 35, 35 J 60, 76 B 10, 76 D 08, 76 E 30, 76 M 30

\section{Introduction}

The aim of this paper is to bridge the gap between Hele-Shaw theory and lubrication approach. Hele-Shaw theory is mainly concerned with flows between two closed fixed flat surfaces while lubrication approach is used for devices with moving surfaces as journal bearing or seals. The preponderance of the shear viscous effects in the lubrication approach prevents the introduction of full-air/full-fluid interface as in the Hele-Shaw theory and induces a new kind of free boundary problems. Relation between this approach and the classical Hele-Shaw problem one's will be clarified.

The plan is as follows:

We consider a first generalization of the Hele-Shaw problem taking squeezing effects and gap geometry into account. As for classical Hele-Shaw problem, using time integration change of variables (see, for example, $[3,16,20,26]$ ), we reformulate the problem as a variational inequality for which the existerice and uiqueness follows from the classical theory of variational inequalities (see, - for example, [11] and [31]). But classical time regularity results are not sufficient to recover the properties of the unknown initial problem. We are able to prove some supplementary regularity results for a time dependent

G. Bayada: Unit. Rech. Ass. (URA) n: 740 du Centre Nat. Rech. Sci. (CNRS), Math. Bãt. 401 Inst. Nat. Sci. Appl: (INSA), F- 69621 Villeurbanne, France

M. Boukrouche: Unit. Rech. Ass. (URA) $n^{\circ} 740$ du Centre Nat. Rech. Sci. (CNRS), Math. Bât. 401 Inst. Nat. Sci. Appl. (INSA), F - 69621 Villeurbanne, France, and Univ. Annaba, Inst. Math. Annaba BP12, Algeria

M. El-A. Talibi: Univ. Caddi-Ayyad, Dep. Math., Marrakesch BP S15, Marocco

ISSN 0232-2064 / \$ 2.50 C Heldermann Verlag Berlin 
vaiational inequality of the first kind, which allows us to recover the properties of the physical unknown and the strong formulation of the initial problem.

If shear effects are to be taken into account, lubrication fluid-mixture approach [21] has to be used, inducing a new family of free boundary problems. The existence proof can be obtained in the same way as [22] and [19] does; uniqueness results are given for a particular case, using a second kind of variational inequality, initially introduced in Brezis [11]. Finally, we prove that the solutions of the weak formualtion of both HeleShaw problems and shearless lubrication problem are the same, establishing Hele-Shaw phenomena as a particular case of lubrication problem on a mathematical basis.

\section{About the physical aspect and strong formulation of the classical Hele-Shaw theory}

Let $\Omega^{e}$ a given volume limited by $S_{1}$ and $S_{2}$, two fixed walls parallel to an $\left(x_{1}, x_{2}\right)$ plane, which are $\varepsilon$ apart, and $\Gamma_{\text {ex }}^{\epsilon}$ lateral vertical boundaries. Initially we assume that fluid occupies a given bounded region $\Omega_{0}^{\epsilon} \subset \Omega^{\varepsilon}$ and is injected through $S_{I}$, a feed hole included in $S_{1}$, with $\partial S_{I}=\Gamma_{I}$. For each $\tau \in(0, T)(T>0)$, the boundary of the unknown region $\Omega^{e}(\tau)$ containing fluid is denoted by $\Gamma^{\prime} \varepsilon(\tau)$ and $\Omega^{\varepsilon}(\tau) \subset \Omega^{\varepsilon}$. We consider the flow of incompressible viscous fluid subject to an exterior force density $F^{\varepsilon}$.

The real three-dimensional problem is for each $\tau \in(0, T)$ to find $\Omega^{\varepsilon}(\tau)$, a velocity field $u^{\varepsilon}(x, \tau)$ and a pressure $p^{\varepsilon}(x, \tau)$ in $\Omega^{\varepsilon}(\tau)$, with $x=\left(x_{1}, x_{2}, x_{3}\right)$ so that

$$
\begin{aligned}
\frac{\partial u^{\varepsilon}}{\partial \tau}-\nu \Delta u^{\varepsilon}+\nabla p^{\varepsilon} & =F^{\varepsilon} & & \text { in } \Omega^{\varepsilon}(\tau) \\
\operatorname{Div} u^{\varepsilon} & =0 & & \text { in } \Omega^{\varepsilon}(\tau) \\
u^{\varepsilon} \cdot N & =\underline{v}^{\varepsilon} \cdot N & & \text { on } \Gamma^{\varepsilon}(\tau) \\
\left(p_{a}-p^{\varepsilon}\right) N_{i}+\nu\left(\frac{\partial u_{i}^{\varepsilon}}{\partial x_{j}}+\frac{\partial u_{j}^{\varepsilon}}{\partial x_{i}}\right) N_{j} & =\frac{\gamma H}{\rho} N_{1} & & \text { on } \Gamma^{\varepsilon}(\tau)(i, j=1,2,3) .
\end{aligned}
$$

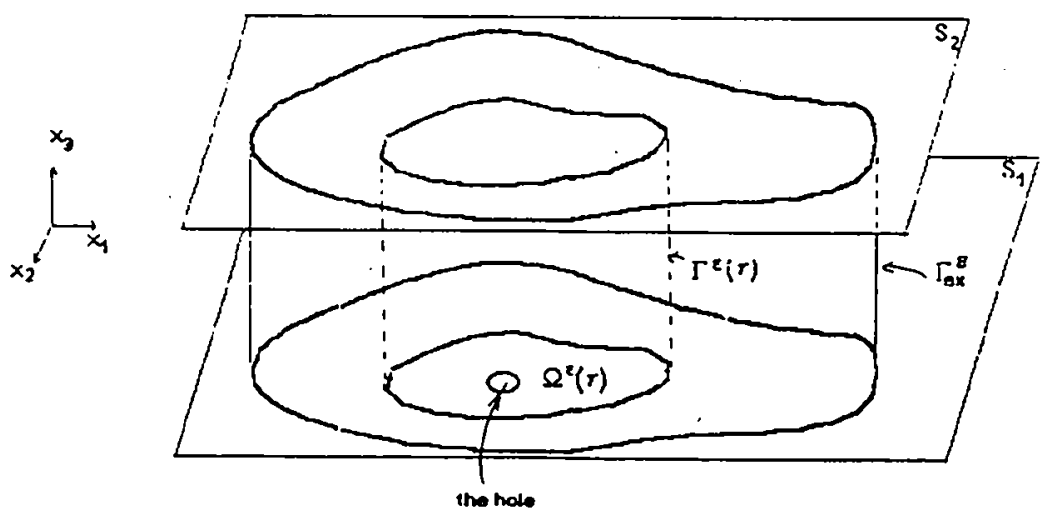

Figure 1: Real 3-D phenomena 
Here $\nu$ is a kinematic viscosity, $N$ is the outward normal on $\Gamma^{\varepsilon}(\tau), \underline{v}^{\epsilon}$ is the velocity of $\Gamma^{e}(\tau), \gamma$ is the coefficient of surface tension, $I H$ is the mean curvature of $\Gamma^{e}(\tau), \rho$ is the density of the fluid and $p_{a}$ is the exterior pressure.

Other boundary conditions are to be given on the velocity and/or the pressure.

At both surfaces $S_{1}$ and $S_{2}$ we have $u^{\varepsilon}=0$, except on the injection hole $S_{1}$ where the fluid is injected with the velocity $u^{e}=\left(u_{1}^{\epsilon}, u_{2}^{\varepsilon}, u_{3}^{\varepsilon}\right)$ such that $u_{1}^{\varepsilon}=u_{2}^{\varepsilon}=0$ and $u_{3}^{\varepsilon}=g\left(x_{1}, x_{2}\right) \varepsilon^{\alpha}$.

Moreover, a supplementary condition, the wetting angle has to be given at the intersection of the free boundary $\Gamma^{\varepsilon}(\tau)$ and the surfaces $S_{1}$ and $S_{2}$. But the knowledge of this angle is very controversy, especially for transient phenomena, as Hele-Shaw one's.

A discussion of equations (1.4) may be found, e.g., in [36: p. 451]. Some results of existence and uniqueness for free boundary Stokes problem appears in $[2,7,8,34]$, but in all of these cases, the free boundary does not touch the external sides, so the problem of the wetting angle does not inters.

In Hele-Shaw model, in view of obtaining a two-dimensional model, it is assumed that the free boundary is vertical (the wetting angle is equal to $\frac{\pi}{2}$ ).

Assuming the surface tension negligible, a dimensional analysis of the preceeding system for small $\varepsilon$ leads to the following conclusions (see [10]):

a) $\alpha$ must be equal to 3 .

b) The dimensionless pressure is independent of $x_{3}$, continuous at the free boundary, and satisfies Hele-Shaw two-dimensional system where $p$ is the conveniently rescaled function for the pressure and $t$ is the conveniently rescaled time:

$$
\begin{aligned}
\Delta p & =0 & & \text { in } \Omega(t) \\
\underline{v} \cdot n & =-\nabla p \cdot n & & \text { on } \Gamma(t) \\
p & =0 & & \text { on } \Gamma(t) \\
\nabla p \cdot n & =W & & \text { on } \Gamma_{\mathrm{I}} \\
p & =0 & & \text { on } \Gamma_{\mathrm{ex}}=\partial \Omega \backslash \Gamma_{\mathrm{I}} \\
p & =0 & & \text { in } \Omega^{0}(t)=\Omega \backslash \Omega(t)
\end{aligned}
$$

where $\Omega(t)$ and $\Gamma(t)$ are the projections on the $\left(x_{1}, x_{2}\right)$-plane of the unknown region $\Omega^{e}(t)$ and his boundary $\Gamma^{\varepsilon}(t)$, respectively, $n$ is the outward normal, $\Gamma_{\mathrm{I}}=\partial S_{\mathrm{I}}$ while $W$ is directly related to the injection flow through $\Gamma_{1}$.

At this stage, no reference has been made to the sign of the pressure, through this assumption plays a major role in the mathematical treatment of that system.

\section{Some mathematical results}

The first analytic treatment of the injection of a fluid appears to go back to Richardson (see [19] and [30]), who formulate the problem as a differential equation for the Riemann mapping function from the unit disc onto $\Omega(t)$, identifying $\mathbb{R}^{2}$ with $\mathbb{C}$ and assuming that $\Omega(t)$ is always simply connected. But no proof of existence or uniqueness of solutions of this differential equation is given in [29] and [30]. However, a partial (small time) 
existence and uniqueness proof for the same differential equation have ben given in [35]. Gustavsson [28] gives a more elementary proof of existence of solutions in the case where $f(\xi)$ is a polynomial or a rational function. In that case the differential equation can be reduced to a finite system of ordinary differential equations in $t$ and this system has a unique solution by standard theory. Here the unknown is an analytic function, no reference has been made to the sign of the pressure. A measure theoretical approach has been introduced by Sakai (see [32] and [33]).

Taking the positivity of the pressure into account and using Baiocchi transformation [3], Gustafsson [26] has investigated the weak (distributional) solutions of the problem, and the related moment problems, whose solutions turn out to be the same as Sakai's solutions. G. Coppoletta [17] considered the weaker problem in which he put the regularized function $\theta: \theta=1$ in $\Omega(t)$ and $0 \leq \theta \leq 1$ in $\Omega \backslash \Omega(t)$, in place of the characteristic function $\chi(t)$ on $\Omega(t)$. With Baiocchi transformation an elliptic variational inequality is obtained. The existence and uniqueness of the solution $(p, \theta)$ of the weaker problem is proved but he was unable to go back to the initial problem $(p, \chi)$.

H. Begehr and R. P. Gilbert have generalized the problem to $\mathbb{R}^{n}$. The injection of fluid takes place at certain discrete points which are given, :as well as the rates of injection at these points. They extend the results obtained by Gustafsson [26] for the Hele-Shaw flows in the plane to flows in $\mathbb{R}^{n}$. In [6] they investigate the problem where the flow rate $u$ is related to the pressure gradient by an anisotropic tensor. In [23] R. P. Gilbert and Wen Guo Chun, using the same analytic treatment as Richardson [29], reduced the problem to an ordinary differential equation describing the solution of a moving boundary problem. S. D. Howison [27]considered cusp development. Other families of problems are obtained by taking Neumann condition on $\Gamma_{\text {ex }}$ (for example, [16], [20] and [24]), or by considering the sucking problem $(W<0)$ (for example, [18] and [25]).

Previous generalizations are mostly mathematical ones. A more physical approach will demonstrate that the pressure must be associated with a particular elliptic operator (in divergence form): the evolutionary Reynolds equation, taking full account of the geometry of the gap and of the.boundary conditions of the surfaces.

\section{First generalization of the Hele-Shaw theory}

3.1 Statement of the problem and notations. We consider the generalization of Hele-Shaw problem taking first squeezing effects and gap geometry into account. The effective gap is denoted by $\varepsilon h(t, x)$ wher $h$ is a smooth function of time and space which does not depend on $\varepsilon$ :

Other conditions satisfied at the fixed and moving boundaries, like (3.2) - (3.5) are obtained in the same way as in the classical Hele-Shaw problem by asymptotic analysis for small $\varepsilon$. The following problem yields:

$\left(\mathbf{P}_{0}^{\prime}\right)$ Find for each $t \in(0, T)$ the pressure $p \in L^{2}\left(0, T ; H^{1}(\Omega)\right.$ ) (where $\Omega$ is the projection on the $\left(x_{1}, x_{2}\right)$-plane of the limited volume $\left.\Omega^{e}\right)$ and the free boundary $\Gamma(t)$, such that

$$
\nabla\left(\frac{h^{3}}{12 \nu} \nabla p\right)=\frac{\partial h}{\partial t} \quad \cdot \text { in } \Omega(t)
$$




$$
\begin{aligned}
-\frac{h^{2}}{12 \nu} \nabla p \cdot n & =\underline{v} \cdot n & & \text { on } \Gamma(t) \\
p & =0 & & \text { on } \Gamma(t) \\
\frac{h^{3}}{12 \nu} \nabla p \cdot n & =W & & \text { on } \Gamma_{1} \\
p & =0 & & \text { on } \Gamma_{\text {ex }}=\partial \Omega \backslash \Gamma_{\mathrm{I}} \\
p & =0 & & \text { in } \Omega^{0}(t):=\Omega \backslash \Omega(t) \\
\Omega(0) & =\Omega_{0} . & &
\end{aligned}
$$

This problem differs from the classical one $\left(\mathrm{P}_{0}\right)$ in nature in that we have Poisson's equation.

3.2 Assumptions and variational formulation. For values of time $t \in(0, T)$, the boundary of the region

$$
\Omega(t)=\{x: t>m(x)\}
$$

containing fluid is denoted by $\Gamma(t)$ and parametrically described by

$$
\Gamma(t)=\{x: S(t, x)=t-m(x)=0\}
$$

Proposition 3.1 (Maximum Principle): Let $p$ a solution of problem $\left(P_{0}^{\prime}\right)$ such that (H.0) $\Gamma(t)$ is sufficiently regular

(H.1) $W \geq 0$ in $\Gamma_{\mathrm{I}}$ and $\frac{\partial h}{\partial t} \leq 0$ in $(0, T) \times \Omega$.

Then

$\left.1^{\circ}\right) p \geq 0$ a.e. in $\Omega \times(0, T)$

$\left.\mathbf{2}^{\circ}\right) \Omega(t) \subset \Omega\left(t^{\prime}\right)$ for any $t<t^{\prime}$.

Proof: From conditions (3.1) and (3.4) and the strong maximum principle we deduce statement $1^{\circ}$. Since $p \geq 0$ in $\Omega(t)$ and $p=0$ outside $\Omega(t)$, then $\frac{\partial p}{\partial n} \leq 0$ on $\Gamma(t)$. Therefore from condition (3.2) we deduce $\underline{v} \cdot n \geq 0$ on $\Gamma(t)$, i.e. statement $2^{\circ}$ follows.

We shall now show how this problem can be reformulated as a variational inequality. To this end, we assume that

(H.2) $h(t, x)=l(t) g(\dot{x})$

(H.3) $h \in C^{1}([0, T] \times \bar{\Omega})$ and, for some $a>0$ and $b .>0, h(t, x) \in(a, b)$ for all. $(t, x) \in[0, T] \times \bar{\Omega}$.

We consider the following change of unknown:

$$
z(t, x)=\frac{1}{12 \nu} \int_{0}^{t} l^{3}(\tau) p(\tau, x) d \tau \quad \text { and } \quad Q(t, x)=\int_{0}^{t} W(\tau, x) d \tau
$$


Remark 3.1: Since $p \geq 0$ in $(0, T) \times \Omega$ and $p=0$ in $\Omega^{0}=\Omega \backslash \Omega(t)$, and because $\Omega(t) \subset \Omega\left(t^{\prime}\right)$ for any $t<t^{\prime}$, then the function $z$ defined by (3.8) also satisfies

$$
z(t) \geq 0 \text { in } \Omega \quad \text { and } \quad z(t)=0 \text { in } \Omega^{0}(t) \text { for any } t \in(0, T) .
$$

Moreover, $z(\cdot, x)$ is increasing for any $x$, so that

$$
\frac{\partial z}{\partial t} \geq 0 \quad \text { in }(0, T) \times \Omega \quad \text { and } \quad \frac{\partial z}{\partial t}=0 \quad \text { in } \Omega^{0}(t) \text { for any } t \in(0, T) .
$$

Formally deriving the differential equation satisfied by $z$, assuming condition (H.0) and the smoothness of $p$, we obtain, using conditions (3.1) - (3.3),

$$
\begin{aligned}
\nabla z & =\frac{1}{12 \nu} \int_{m(x)}^{\ell} l^{3}(\tau) \nabla p(\tau, x) d \tau-\frac{1}{12 \nu} l^{3} m(x) p(m(x), x) \nabla m(x) \\
& =\frac{1}{12 \nu} \int_{m(x)}^{t} l^{3}(\tau) \nabla p(\tau, x) d \tau \quad\left((t, x) \in(m(x), T) \times\left(\Omega \backslash \Omega_{0}\right)\right)
\end{aligned}
$$

and

$$
\begin{aligned}
\operatorname{div}\left(g^{3} \nabla z\right) & =\int_{m(x)}^{t} \operatorname{div}\left(\frac{h^{3}}{12 \nu} \nabla p(\tau, x)\right) d \tau-\frac{1}{12 \nu} h^{3}(m(x), x) \nabla p(m(x), x) \nabla m(x) \\
& =\int_{m(x)}^{t} \frac{\partial h}{\partial t}(\tau, x) d \tau+h(m(x), x) \\
& =h(t, x) \quad\left((t, x) \in(m(x), T) \times\left(\Omega \backslash \Omega_{0}\right)\right)
\end{aligned}
$$

and

$$
\begin{aligned}
\operatorname{div}\left(g^{3} \nabla z\right) & =\int_{0}^{t} \operatorname{div}\left(\frac{h^{3}}{12 \nu} \nabla p(\tau, x)\right) d \tau=\int_{0}^{t} \frac{\partial h}{\partial t}(\tau, x) d \tau \\
& =h(t, x)-h(0, x)=h(t, x)-h_{0}(x) \quad\left((t, x) \in(0, T) \times \Omega_{0}\right) .
\end{aligned}
$$

Denoting by $\chi_{0}$ the characteristic function of $\Omega_{0}$, we obtain that $z$ solves the linear complementarity problem

$$
\begin{aligned}
\left(-\operatorname{div}\left(g^{3} \nabla z\right)-\chi_{0} h_{0}(x)+h(t, x)\right) & \geq 0 \\
z & \geq 0 \quad \text { in } \Omega \\
\left(-\operatorname{div}\left(g^{3} \nabla z\right)-\chi_{0} h_{0}(x)+h(t, x)\right) z & =0
\end{aligned}
$$


with boundary conditions

$$
g^{3} \frac{\partial z}{\partial n}=Q \quad \text { on } \Gamma_{\mathrm{I}}, \quad z=0 \quad \text { on } \Gamma_{\mathrm{ex}}, \quad z=\frac{\partial z}{\partial n}=0 \quad \text { on } \Gamma(t) .
$$

Let $K$ be the closed convex subset of $H^{1}(\Omega)$ defined by

$$
K=\{\varphi \in \mathbb{W}: \varphi \geq 0 \text { a.e. in } \Omega\} \text { with } \mathbb{W}=\left\{\varphi \in H^{1}(\Omega): \varphi=0 \text { on } \Gamma_{\mathrm{ex}}\right\}
$$

Then problem (3.9), (3.10) can be written as the following variational problem:

$\left(\mathbf{P}_{1}^{\prime}\right)$ Find $z(t) \in K$ for each $t \in(0, T)$ such that

$$
\int_{\Omega} g^{3} \nabla z \nabla(\varphi-z) \geq \int_{\Omega}\left(\chi_{0} h_{0}(x)-h(t, x)\right)(\varphi-z)+\int_{\Gamma_{\mathrm{I}}} Q(\varphi-z)
$$

for all $\varphi \in K$.

For each $t \in(0, T)$, the existence and uniqueness of a solution $z \in L^{\infty}\left(0, T ; H^{1}(\Omega)\right)$ of problem $\left(\mathrm{P}_{1}{ }_{1}\right)$ follows from the classical theory of variational inequalities (see [11] and [31]). As already mentioned this regularity is not sufficient to recover the initial properties of $p$ given in problem ( $\left.\mathrm{P}_{0}^{\prime}\right)$.

:To go back to the initial problem $\left(\mathrm{P}_{0}^{\prime}\right)$, supplementary results are needed for the properties of the time dependent variational inequality $\left(\mathrm{P}_{1}^{\prime}\right)$.

3.3 Some regularity results for a time dependent variational inequality of the first kind. Let $F$ and $G$ such that

(H.E) $F \in C^{1}\left(0, T ; H^{-1}(\Omega)\right) \cap L^{\infty}(\Omega \times(0, T))$ and $G \in C^{1}(\bar{\Omega} \times[0, T])$

and let us consider the following problem:

$\left(\mathbf{P}_{2}\right)$ Find $v(t) \in K$ for each $t \in(0, T)$ such that $a(v, \varphi-v) \geq(F, \varphi-v)+\langle G ; \varphi-v\rangle$ for all $\varphi \in K$ and $v(0)=0$.

Here $K$ and $W$ are defined in $(3.11), a(\cdot, \cdot)$ is the bilinear continuous and coercive form on $W$ defined by

$$
a(v, \varphi)=\sum_{i, j=1}^{2} \int_{\Omega} a_{i j} \frac{\partial v}{\partial x_{i}} \frac{\partial \psi}{\partial x_{j}} d x \quad\left(\psi \ddot{\epsilon} H^{1}(\Omega)\right)
$$

with $a_{i j}=a_{j i} \in C^{2}(\bar{\Omega})$ and $A$ the associated second order operator

$$
A=-\sum_{i, j=1}^{2} \frac{\partial}{\partial x_{i}}\left(a_{i j} \frac{\partial}{\partial x_{j}}\right)
$$


$(\cdot, \cdot)$ denotes the usual inner product

$$
(F, \Psi)=\int_{\Omega} F \Psi d x \quad\left(\psi \in L^{2}(\Omega)\right)
$$

in $L^{2}(\Omega)$ and $\langle\cdot$,$\rangle defines the duality product$

$$
\langle G, \Psi\rangle=\int_{\Gamma_{1}} G \Psi d \sigma \quad\left(\Psi \in L^{2}\left(\Gamma_{1}\right)\right)
$$

in $L^{2}\left(\Gamma_{\mathrm{I}}\right)$ with $\Gamma_{\mathrm{I}} \cap \Gamma_{\mathrm{ex}}=\emptyset$.

Existence and uniqueness for $v$ satisfying problem $\left(P_{2}\right)$ is obvious. In order to study the time regularity of $v$, we consider the following penalised problem (see [31: p. 276]):

$\left(\mathbf{P}_{\mathbf{2}}^{e}\right) \quad v_{\varepsilon}=0$ on $\Gamma_{\mathrm{ex}}$ and $\sum_{i, j=1}^{2} a_{i j} \frac{\partial v_{\varepsilon}}{\partial x_{i}} \cos \left(n, x_{j}\right)=G$ on $\Gamma_{\mathrm{I}}$

where $e=e(t, x)$ is a parameter function.

Let us introduce the assumption

( $\mathbf{H ~ e )}$

$$
\begin{aligned}
& e \in C^{1}\left(0, T ; H^{-1}(\Omega)\right) \cap L^{\infty}(\Omega \times(0, T)) \\
& e \geq 0, \quad \frac{\partial e}{\partial t} \leq 0, \quad F+e \geq 0, \quad \frac{\partial(F+e)}{\partial t} \geq 0 .
\end{aligned}
$$

For example, $e(t, x)=\left(1-\chi_{0}\right) h(t, x), F(t, x)=\chi_{0} h_{0}(x)-h(t, x)$ for some regular function $h_{0}=h(0, x)$ and $H_{\varepsilon}$ is an approximation of the Heaviside graph $H$ as, for example,

$$
H_{\varepsilon}(s)= \begin{cases}1 & \text { if } s \geq \varepsilon \\ s / \varepsilon & \text { if } 0 \leq s \leq \varepsilon \\ 0 & \text { if } s \leq 0\end{cases}
$$

In the following we need also the supplementary assumptions

(H G) $\quad G \geq 0$ and $\frac{\partial G}{\partial t} \geq 0$

(H G F) $\frac{\partial G}{\partial t} \geq G$ and $\left(\frac{\partial F}{\partial t}+\frac{\partial e}{\partial t}-F-e\right) \geq 0$.

Theorem 3.1. Assuming (HE), (He) and (HG), there exists a unique solution $v_{\varepsilon} \in K$ to problem $\left(P_{2}^{\varepsilon}\right)$, for every $\varepsilon>0$, and $v_{\varepsilon}(t)$ strongly converges in $H^{1}(\Omega)$, as $\varepsilon$ tend to zero, to the solution $v(t)$ of problem $\left(P_{2}\right)$, for each $t \in(0, T)$, and the error estimate

$$
\left\|v-v_{e}\right\|_{H^{1}(\Omega)} \leq C \sqrt{\varepsilon} \quad \text { where } C=\frac{1}{\inf a_{i j}} \int_{\Omega} e d x
$$

is true.

Proof. We have, for $\varphi \in \mathbb{W}$,

$$
a\left(v_{\varepsilon}, \varphi\right)+\left(e H_{\varepsilon}\left(v_{\varepsilon}\right), \varphi\right)=(F+e, \varphi)+\langle G, \varphi\rangle
$$

as $F+e \geq 0$ and $G \geq 0$. Multiplying (3.13) by $v_{\varepsilon}^{-}$leads to $v_{\varepsilon}^{-}=0$, i.e. $v_{e} \in K$. Taking $\varphi=v-v_{\epsilon}$ in (3.13) $\varphi=v_{e}$ in problem $\left(P_{2}\right)$ and adding we deduce the statement. 
Supplementary regularity results are given in the following

Theorem 3.2. Assuming ( $\mathrm{HE}),(\mathrm{He})$ and $(\mathrm{H} \mathrm{G})$, the solution $v$ of problem $\left(P_{2}\right)$ is such that

$$
\begin{aligned}
v & \in W^{1, \infty}\left(0, T ; H^{1}(\Omega)\right) \\
\frac{\partial v}{\partial t} & \geq 0 \text { a.e. on }[0, T]
\end{aligned}
$$

is true.

Proof. Let $t, s \in[0, T]$. Putting $W=v_{e}(t)-v_{e}(s)$ and subtracting (3.13) for $s$ from (3.13) for $t$ we obtain.

$$
\begin{aligned}
a(W, \varphi)+ & \left(e(t) H_{\varepsilon}\left(v_{e}\right)\right)-e(s) H_{\varepsilon}\left(V_{\varepsilon}(s), \varphi\right) \\
& =(F(t)+e(t)-F(s)-e(s), \varphi)+\langle G(t)-G(s), \varphi\rangle
\end{aligned}
$$

for all $\varphi \in \mathbb{V}$. Choosing $\varphi \in \mathbb{W}$ in (3.16) we have

$$
\begin{aligned}
a(W, W)+ & \left(e(t) H_{\varepsilon}\left(v_{\varepsilon}(t)-H_{\varepsilon}\left(\dot{v}_{e}(s)\right), W\right)+\left((e(t)-e(s)) H_{\varepsilon}\left(z_{\varepsilon}(s)\right), W\right)\right. \\
= & ((F+e)(t)-(F++e)(s), \mathbb{W})+\langle G(t)-G(s), W\rangle
\end{aligned}
$$

and, as

$$
\left(H_{\varepsilon}\left(v_{\varepsilon}(t)\right)-H_{\varepsilon}\left(v_{\varepsilon}(s)\right)\right) W \quad \text { and } \quad e \geq 0
$$

we have

$$
\begin{gathered}
a(\mathbb{W}, W) \leq|t-s|\left(2\left\|\left|\frac{e(t)-e(s)}{t-s}\right|+\left|\frac{F(t)-F(s)}{t-s}\right|\right\|_{L^{2}(\Omega)} .\right. \\
\left.+\left\|\frac{G(t)-G(s)}{t-s}\right\|_{L^{2}\left(\Gamma_{I}\right)}\right)\|W\|_{H^{1}(\Omega)}
\end{gathered}
$$

and from assumptions (H.E) and ( $\mathrm{He}$ ) we get

$$
\left\|v_{\varepsilon}(t)-v_{\varepsilon}(s)\right\|_{H^{1}(\Omega)} \leq C|t-s| \quad \cdot((t, s) \in[0, T] \times[0, T])
$$

where

$$
C=\frac{c_{1}}{\inf a_{i j}} \sup _{\Omega \times[0, T]}\left(2\|e\|_{C^{1}\left(0, T ; H^{-1}(\Omega)\right)}+\|\dot{F}\|_{C^{1}\left(0, T ; H^{-1}(\Omega)\right)}+\|G\|_{C^{1}(\bar{\Omega} \times[0, T])}\right)
$$

with a constant $c_{1}$ depending only of $\Omega$.

While passing to the limit on $\varepsilon$ in (3.7) we obtain

$$
\|v(t)-v(s)\|_{H^{\mathrm{l}}(\Omega)} \leq C|t-s| \text { for all }(t, s) \in[0 ; T] \times[0, T] .
$$

We deduce (3.14) as a consequence of (3.18). From (3.17) if we put $u_{e}=\partial v_{\varepsilon} / \partial t$ so $u_{e}$ is a solution of the problem

with boundaries conditions

$$
A u_{e}+e H_{e}^{\prime}\left(v_{e}(t)\right) u_{e}=\frac{\partial F}{\partial t}-\frac{\partial e}{\partial t} H_{e}\left(v_{e}(t)\right)+\frac{\partial e}{\partial t}
$$

$$
u_{\varepsilon}=0 \text { on } \Gamma_{\mathrm{ex}} \text { and } \sum_{i, j=1}^{2} a_{i j} \frac{\partial u_{\varepsilon}}{\partial x_{i}} \cos \left(n, x_{j}\right)=\frac{\partial G}{\partial t} \text { on } \Gamma_{\mathrm{I}} \text {. }
$$

Multiplying (3.19) by $u_{e}^{-}$, using assumptions (He) and (H G) and the fact that $H_{\varepsilon}^{\prime} \geq 0$, we obtain $u_{\varepsilon}^{\prime}=0$, i.e. $\frac{\partial v_{k}}{\partial t} \geq 0$, and, as $\frac{\partial v_{k}}{\partial t} \rightarrow \frac{\partial v}{\partial t}$ weakly in $L^{2}\left(0, T ; H^{1}\right)$, then $\frac{\partial v}{\partial t} \geq 0$ a.e. in $L^{2}\left(0, T ; H^{1}\right)$. 
Theorem 3.3. Assuming ( $\mathrm{HE}),(\mathrm{He})$ and ( $\mathrm{H} \mathrm{F} \mathrm{G})$ we have

$$
\{x \in \Omega: v(t, x)>0\}=\left\{x \in \Omega: \frac{\partial v}{\partial t}(t, x)>0\right\} \text { a.e. on }(0, T] .
$$

Proof. From the penalized problem $\left(\mathrm{P}_{2}^{\epsilon}\right)$, we have

$$
A v_{\varepsilon}=-e H_{\varepsilon}\left(v_{\varepsilon}\right)+F+e \text { in } H^{-1}(\Omega)
$$

and by derivation we get

$$
A\left(\frac{\partial v_{\varepsilon}}{\partial t}\right)=-\frac{\partial e}{\partial t} H_{\epsilon}\left(v_{\varepsilon}\right)-e H_{\varepsilon}^{\prime}\left(v_{\varepsilon}\right) \frac{\partial v_{\varepsilon}}{\partial t}+\frac{\partial e}{\partial t}+\frac{\partial F}{\partial t} \quad \text { in } H^{-1}(\Omega)
$$

Putting $U_{\varepsilon}=\frac{\partial v_{\varepsilon}}{\partial t}-v_{\varepsilon}$ we obtain

$$
A U_{\epsilon}=\frac{\partial c}{\partial t}\left(1-H_{\varepsilon}\left(v_{\varepsilon}\right)\right)-e H_{\varepsilon}\left(v_{\varepsilon}\right) \frac{\partial v_{\varepsilon}}{\partial t}+\frac{\partial F}{\partial t}+e H_{\varepsilon}\left(v_{\varepsilon}\right)-F-e .
$$

Using the Green formula we gain

$$
\begin{aligned}
a\left(U_{\varepsilon}, U_{\varepsilon}^{-}\right)= & \left\langle\frac{\partial G}{\partial t}-G, U_{\varepsilon}^{-}\right\rangle+\left(\left(e-\frac{\partial e}{\partial t}\right) H_{\varepsilon}\left(v_{\varepsilon}\right), U_{\varepsilon}^{-}\right) \\
& -\left(e H_{\varepsilon}^{\prime}\left(v_{\varepsilon}\right) \frac{\partial v_{\varepsilon}}{\partial t}, U_{\varepsilon}^{-}\right)+\left(\frac{\partial F}{\partial t}+\frac{\partial e}{\partial t}-F-e, U_{\varepsilon}^{-}\right) .
\end{aligned}
$$

From assumption (H.E) we have $\left(e-\frac{\partial e}{\partial t}\right) \geq 0$, thus

$$
\begin{aligned}
a\left(U_{\varepsilon}, U_{\varepsilon}^{-}\right) \geq\langle & \left.\frac{\partial G}{\partial t}-G, U_{\varepsilon}^{-}\right\rangle-\left(e H_{\varepsilon}^{\prime}\left(v_{\varepsilon}\right) \frac{\partial v_{\varepsilon}}{\partial t}, U_{\varepsilon}^{-}\right) \\
& +\left(\frac{\partial F}{\partial t}+\frac{\partial e}{\partial t}-F-e, U_{\varepsilon}^{-}\right) .
\end{aligned}
$$

Using now assumption ( $\mathrm{H} \mathrm{G} \mathrm{F}$ ), the inequality

$$
a\left(U_{\varepsilon}^{-}, U_{\varepsilon}^{-}\right) \leq\left(e H_{\varepsilon}^{\prime}\left(v_{\varepsilon}\right) \frac{\partial v_{\varepsilon}}{\partial t}, U_{\varepsilon}^{-}\right)=\int_{\Sigma} e H_{\varepsilon}^{\prime}\left(v_{\varepsilon}\right) \frac{\partial v_{\varepsilon}}{\partial t} U_{\varepsilon}^{-}
$$

is obtained where $\Sigma=\left[0 \leq \frac{\partial v_{\varepsilon}}{\partial t}<v_{\varepsilon}<\varepsilon\right]$. In $\Sigma$ we have

$$
H_{\varepsilon}^{\prime}\left(v_{\varepsilon}(t)\right) \leq \frac{1}{\epsilon} \quad \text { and } \quad U_{\varepsilon}^{-}=v_{\varepsilon}-\frac{\partial v_{\varepsilon}}{\partial t} \leq \varepsilon
$$

Therefore $U_{\varepsilon} \frac{\partial v_{\varepsilon}}{\partial t} \leq \varepsilon^{2}$. So $a\left(U_{\varepsilon}^{-}, U_{\varepsilon}^{-}\right) \leq C \varepsilon$. And from the coerciveness of the bilinear form $a(\cdot, \cdot)$ we deduce that $U_{\varepsilon}^{-} \rightarrow 0$ in $L^{2}(\Omega)$, but

$$
\left(\frac{\partial v_{\varepsilon}}{\partial t}-v_{\varepsilon}\right)^{-} \rightarrow\left(\frac{\partial v}{\partial t}-v\right)^{-} \quad \text { weakly in } L^{2}(\Omega)
$$

Therefore, $\left(\frac{\partial v}{\partial t}-v\right)^{-}=0$ a.e. in $L^{2}(\Omega)$. Moreover, as $\frac{\partial v}{\partial t} \geq 0$ a.e. on $(0, T)$ (see Theorem $3.2)$, if $v(t, x)=0$, we deduce that $v(\tau, x)=0$ for all $\tau \in(0, t]$. Then $\frac{\partial v}{\partial t}(\tau, x)=0$ for all $\tau \in(0, t]$, i.e. $\frac{\partial v}{\partial t}=0$. 
3.4 Application to the generalized Hele-Shaw problem $\left(\mathbf{P}_{0}^{\prime}\right)$. In the following, we will show how the preceding results in Subsection 3.3 can be used to give regularity results for the generalized Hele-Shaw problem $\left(\mathrm{P}_{0}^{\prime}\right)$.

Theorem 3.4. If the function $h$ satisfies the assumptions (H.2) and (H.3), $\frac{\partial h}{\partial t} \leq 0$ in $(0, T) \times \Omega$, and if the function $Q \in C^{1}([0, T] \times \Omega)$ satisfies $Q \geq 0$ and $\frac{\partial Q}{\partial t} \geq 0$, then the unique solution $z$ of the variational problem $\left(\mathrm{P}_{1}^{\prime}\right)$ is such that

$$
z \in W^{1, \infty}\left(0, T ; H^{1}(\Omega)\right) \cap L^{\infty}\left(0, T ; H^{2}(\Omega) \cap \mathbb{W}\right) \quad \text { and } \quad \frac{\partial z}{\partial t} \geq 0 \text { a.e. }
$$

Moreover, assuming (H.0), $W \geq Q$ and $\left(\frac{\partial h}{\partial t}+h_{0}-h\right) \chi_{0} \leq 0$ we deduce that $12 \nu l^{-3} \frac{\partial z}{\partial t}$ satisfies the initial problem $\left(\mathrm{P}_{0}^{\prime}\right)$, where $\Omega(t)=\{x \in \Omega: z(t, x)>0\}$ for any $t>0$.

Proof. Let $F=\chi_{0} h_{0}-h, G=Q, a(z, \Psi)=\int_{\Omega} g^{3} \nabla z \nabla \Psi$ and $e=\left(1-\chi_{0}\right) h$. As $F+e=\left(h-h_{0}\right) \chi_{0} \in L^{2}(\Omega)$, we deduce, from the classical theory of elliptic equations (see [1]) that the solution $v_{e}$ of the problem $\left(\mathrm{P}_{2}^{\epsilon}\right)$ belongs to $H^{2}(\Omega)$ and there exists a constant $C$ depending on $\Omega$ such that

$$
\left\|v_{\varepsilon}(t)\right\|_{H^{2}(\Omega)} \leq C\left(2\|e\|_{C^{1}\left(0, T ; L^{2}(\Omega)\right)}+\|F\|_{C^{1}\left(0, T_{i} L^{2}(\Omega)\right)}+\|\tilde{G}\|_{C^{1}\left(0, T ; H^{1}(\Omega)\right)}\right)
$$

for any lift $\tilde{G}$ of $G$ in $H^{1}(\Omega)$ such that $\tilde{G}=Q$ on $\Gamma_{\mathrm{I}}$ and $\tilde{G}=0$ on $\Gamma_{\mathrm{ex}}$, which induces a weak convergence in $L^{\infty}\left(0, T ; H^{2}(\Omega)\right)$ of a subsequence of $v_{\varepsilon}$.

The previous choice of $f, G$ and $e$ obviously satisfies the assumptions ( $\mathrm{He}$ ), ( $\mathrm{HE}$ ) and $(\mathrm{H} \mathrm{G})$ and allows to apply Theorem 3.1. So (3.12) is valid. So $v_{e}$ strongly converges to $v$ in $L^{\infty}\left(0, T ; H^{1}(\Omega)\right)$, which is also the weak limit of $v_{e}$ in $L^{\infty}\left(0, T ; H^{2}(\Omega)\right)$, and using Theorem 3.2 the first part of the present theorem follows, in particular for the unique solution $z$ of the problem $\left(\mathrm{P}_{1}^{\prime}\right)$.

To prove the second part, we remark first that $\Omega(t)$ defined above is a well defined open set as $z(t) \in H^{2}(\Omega)$. Taking first $\varphi=z \pm \varepsilon \Psi$ with $\Psi$ in $D(\Omega(t))$ - the usual space of $C^{\infty}$ functions : with compact support in $\Omega(t)$ - in the variational problem $\left(\mathrm{P}_{1}^{\prime}\right)$, we obtain

$$
a(z, \Psi)=(f, \Psi)+\langle Q, \Psi\rangle \text { in } \Omega(t) .
$$

Using the Green formula, we have

$$
-\operatorname{div}\left(g^{3} \nabla z\right)=f=\chi_{0} h_{0}-h \quad \text { in } \Omega(t) .
$$

Putting $Q=\Omega \times(0, T), Q^{+}=\Omega(t) \times(0, T)$ and $Q^{0}=\mathbb{Q} \backslash \mathbb{Q}^{+}$we have

$$
-\operatorname{div}\left(g^{3} \nabla z\right)=f=\chi_{0} h_{0}-h \quad \text { in } \mathbb{Q}^{+}
$$

and by derivation we gain the equation (3.1) in $Q^{+}$

$$
-\operatorname{div}\left(g^{3} \nabla\left(\frac{\partial z}{\partial t}\right)\right)=-\operatorname{div}\left(\frac{h^{3}}{12 \nu} \nabla\left(12 v l^{-3} \frac{\partial z}{\partial t}\right)\right)=-\frac{\partial h}{\partial t} \cdot \operatorname{in} \dot{Q}^{+} .
$$


Taking $\varphi \in D(\mathbb{Q})$ and using the duality $(\cdot, \cdot\rangle$ between the spaces $D^{\prime}(\mathbb{Q})$ and $D(Q)$, we have

$$
\begin{aligned}
\left\langle-\operatorname{div}\left(g^{3} \nabla\left(\frac{\partial z}{\partial t}\right)\right), \varphi\right\rangle & =\left\langle g^{3} \nabla\left(\frac{\partial z}{\partial t}\right), \nabla \varphi\right\rangle \\
& =\int_{Q^{+}} g^{3} \nabla\left(\frac{\partial z}{\partial t}\right) \nabla \varphi \\
& =\int_{Q^{+}}-\operatorname{div}\left(g^{3} \nabla\left(\frac{\partial z}{\partial t}\right)\right) \varphi+\int_{\Sigma} g^{3} \frac{\partial}{\partial n}\left(\frac{\partial z}{\partial t}\right) \varphi
\end{aligned}
$$

where $\Sigma=U_{t \in(0, T)} \Gamma(t)$ and

$$
\left\langle-\frac{\partial(h \chi)}{\partial t}, \varphi\right\rangle=\int_{Q^{+}} h \frac{\partial \varphi}{\partial t}=-\int_{Q^{+}} \frac{\partial h}{\partial t} \varphi+\int_{\Sigma} h \varphi \cos (n, t) .
$$

And using the above equation we gain

$$
\int_{\Sigma} g^{3} \frac{\partial}{\partial n}\left(\frac{\partial z}{\partial t}\right) \varphi=\int_{\Sigma} h \varphi \cos (n, t)
$$

Therefore

$$
g^{3} \frac{\partial}{\partial n}\left(\frac{\partial z}{\partial t}\right)=h \cos (n, t) \quad \text { thus } \quad \frac{h^{3}}{12 \nu} \frac{\partial}{\partial n}\left(12 \nu l^{-3} \frac{\partial z}{\partial t}\right)=-h \underline{v} n
$$

on $\Sigma$ and so (3.2) follows. By time derivation of the first equality in (3.10) and of (3.8) we gain (3.4). Finally (3.3), (3.5) and (3.6) follow from Theorem 3.3.

Remark 3.2. For the classical Hele-Shaw problem $\left(\mathrm{P}_{0}\right), h$ and $W$ have constant values. Then from Theorem 3.2, the unique solution $z$ of the variational problem

$\left(\mathbf{P}_{1}\right)$ Find $z(t) \in K$ for each $t \in(0, T)$ such that

$$
\int_{\Omega} \nabla z \nabla(\varphi-z) \geq \int_{\Omega}\left(\chi_{0}-1\right)(\varphi-z)+\int_{\Gamma_{1}} t W(\varphi-z) \quad(\varphi \in K)
$$

satisfies the following properties:

$$
\begin{aligned}
& z \in W^{1, \infty}\left(0, T ; H^{1}(\Omega)\right) \cap L^{\infty}\left(0, T ; H^{2}(\Omega) \cap \mathbb{V}\right) \\
& z(0)=0, \quad z \geq 0, \quad \frac{\partial z}{\partial t} \geq 0 \text { a.e. }
\end{aligned}
$$

Moreover, assuming (H.0), $W \geq 0$ and $t \leq 1$ we deduce that $\frac{\partial z}{\partial t}$ satisfies the initial problem: $\left(\mathrm{P}_{0}\right) /(1.5)-(1.9)$, where $\Omega(t)=\{x \in \Omega: z(t, x)>0\}$ for $t>0$. 


\section{Flow between closed surfaces with shear effects: The lubrication approach}

In this case, as for example in a journal bearing, the surface $S_{1}$ has a horizontal velocity, while $S_{2}$ has only a vertical motion so that shear effects are propitious. It is not possible to assure the assumption of a vertical free boundary which would be attached, one of the ends to $S_{1}$, and the other end to $S_{2}$.

Experimental results [21: p. 15] makes the occurence of two distinct areas, one full of fluid which will be named $\Omega^{e}(\tau)$ (where Stokes equation is valid and $p>0$ ), the other $\Omega^{e} \backslash \Omega^{e}(\tau)$ is the cavitated zone, where the pressure is constant $(p=0)$ and appears to be a mixture of fluid and air.

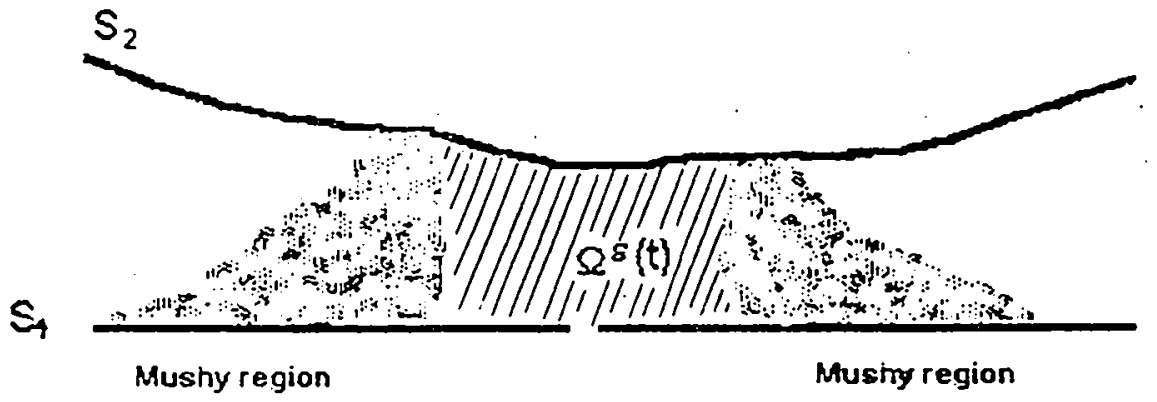

Figure 2: The cavitation phenomenon

Two approachs have been used to cope with this phenomena. One of them [21: p. 37) homogenizes the phenomena and considers it as a two-dimensional phenomena by introducing $\theta$, the lubricant concentration. The other one [14] takes full account of the three-dimensional character of this phenomena, with the appearance of bubbles of air and introduces in $\Omega^{\varepsilon} \backslash \Omega^{\epsilon}(\tau)$ the relative height as a supplementary unknown. We use here the first approach. Both approaches lead to the same mathematical problem.

By definition, the value of $\theta$ is one on $\Omega^{e}(\tau)$ and lies between zero and one in the mushy region. The incompressibility condition (1.2) of the fluid is replaced by the mass conserving condition

$$
\frac{\partial \theta}{\partial \tau}+\operatorname{div}\left(\theta u^{e}\right)=0
$$

which is valid on the whole $\Omega^{e}$. Boundary conditions for the velocity are

on $S_{1}: \quad u_{i}^{e}=\frac{1}{2} g_{i}(i=1,2), u_{3}^{e}=0$ on $S_{1} \backslash S_{\mathrm{I}}$ and $u_{3}^{e}=\varepsilon^{\alpha} g_{3}$ on $S_{\mathrm{I}}$

on $S_{2}: \quad u_{i}^{e}=0(i=1,2)$ and $u_{3}^{e}=\frac{\partial H}{\partial r}$

where $g_{i}$ are known functions of $\tau, x_{1}$ and $x_{2}$.

As in the previous subsection, an asymptotic analysis leads [10] to the following limit system as $\varepsilon$ goes to zero, with the assumption that $\alpha=3$ and $\tau=t$ and introducing 
$y=\frac{x_{3}}{e}:$

$$
\begin{aligned}
\frac{\partial p}{\partial x_{i}} & =\nu \frac{\partial^{2} u_{i}}{\partial y^{2}}(i=1,2) & & \text { in } \Omega(t) \\
\frac{\partial p}{\partial y} & =0 & & \text { in } \Omega(t) \\
\nu \frac{\partial^{2} u_{i}}{\partial y^{2}} & =0 \text { and } p=0 & & \text { in } \Omega \backslash \Omega(t)
\end{aligned}
$$

where $p$ and $u$ are rescaled functions. As $p$ is independent of $y$, integrating $(4.2)_{a}$ with respect to $y$ and taking into account the boundary conditions we obtain (see [15: p. 25])

$$
u_{i}=\frac{1}{2 \nu} y(y-h) \frac{\partial p}{\partial x_{i}}+g_{i} \frac{y}{h}
$$

Integrating $u_{i}$ with respect to $y$ between 0 and $h$, and using the mass conserving condition (4.1), we have, for any $\left(x_{1}, x_{2}\right) \in \Omega \backslash S_{1}$,

$$
\frac{\partial}{\partial t}\left(\int_{0}^{h} \theta d y\right)-\theta \frac{\partial h}{\partial t}+\sum_{i=1}^{2} \frac{\partial}{\partial x_{i}}\left(\theta \int_{0}^{h} u_{i} d y\right)+\left.\theta u_{3}\right|_{y=h}=0
$$

as

$$
\left.u_{3}\right|_{y=h}=\frac{\partial h}{\partial t} \quad \text { and } \quad \int_{0}^{h} u_{i} d y=-\frac{h^{3}}{12 \nu} \frac{\partial p}{\partial x_{i}}+g_{i} \frac{h}{2} .
$$

Then from (4.4) the $2 D$ Reynolds equations yields

$$
\operatorname{div}\left(\frac{h^{3}}{12 \nu} \nabla p\right)=\frac{\partial(\theta h)}{\partial t}+\operatorname{div}(\theta h V)
$$

where $V=\frac{1}{2}\left(g_{1}\left(t, x_{1}, x_{2}\right), g_{2}\left(t, x_{1}, x_{2}\right)\right)$. Finally we get the following strong formulation:

$$
\begin{aligned}
\operatorname{div}\left(\frac{h^{3}}{12 \nu} \nabla p\right) & =\frac{\partial h}{\partial t}+\operatorname{div}(h V), \theta=1 . & & \text { in } \quad \dot{\Omega}(t) \\
\frac{\partial(\theta h)}{\partial t}+\operatorname{div}(\theta h V) & =0 & & \text { in } \Omega^{0}(t)=\Omega \backslash \Omega(t) \\
\frac{h^{3}}{12 \nu} \frac{\partial p}{\partial n} & =h(1-\theta)(V-\underline{v}) \cdot n & & \text { on } \Gamma(t) \\
p & =0 & & \text { on } \Gamma(t) \\
W & =\frac{h^{3}}{12 \nu} \frac{\partial p}{\partial n}-h \theta V \cdot n . & & \text { on } \Gamma_{I} \\
p & =0 & & \text { on } \Gamma=\partial \Omega \backslash \Gamma_{I} \\
p(1-\theta) & =0 & & \text { in } \\
\theta & =\theta_{0} & & \text { at } t=0 .
\end{aligned}
$$


Before stating a weak formulation of this problem $\left(P_{3}\right)$, we define

$$
\begin{aligned}
& \mathbb{Q}=\Omega \times(0, T) \\
& \Sigma_{\mathrm{I}}=\Gamma_{\mathrm{I}} \times(0, T) \\
& \Omega_{t}=\Omega \times\{t\} \text { for } t \in[0, T] \\
& \Sigma_{\mathrm{ex}}=\Gamma_{\mathrm{ex}} \times(0, T) \\
& E=\left\{\varphi \in H^{l}(\mathbb{Q}): \varphi=0 \text { on } \Sigma_{\mathrm{ex}}, \text { at } t=0 \text { and } t=T\right\}
\end{aligned}
$$

and we assume that

(H.5) $V \in\left(H^{1}(\mathcal{Q}) \times H^{1}(\mathbb{Q})\right) \cap\left(L^{\infty}(\mathbb{Q}) \times L^{\infty}(\mathbb{Q})\right)$.

The weak formulation of the problem $\left(\mathrm{P}_{3}\right)$ is:

$\left(\mathbf{P}_{4}\right)$ Find a pair $(p, \theta)$ satisfying

$$
\begin{array}{ll}
p \in L^{2}\left(0, T ; H^{1}(\Omega)\right), \quad \theta \in L^{\infty}(Q) \cap H^{1}\left(0, T ; H^{-1}(\Omega)\right) & \\
0 \leq \theta \leq 1, \quad p(1-\theta)=0 & \text { a.e in } Q \\
p=0 & \text { on } \Sigma_{\text {ex }} \\
\int_{Q} \theta h \frac{\partial \varphi}{\partial t}-\frac{1}{12 \nu} \int_{Q} h^{3} \nabla p \nabla \varphi+\int \theta h V \nabla \varphi+\int_{Q} W \varphi=0 & \forall \varphi \in E \\
\left.\theta\right|_{t=0}=\theta_{0} & \text { in } H^{-1}(\Omega)
\end{array}
$$

In [22] and [19] Gilardi and El-Alaoui studied a problem very similar to the problem $\left(\mathrm{P}_{4}\right)$. Main difference being related with the boundary conditions. They proved the existence of a solution for this kind of problem by way of an approximation by an elliptic problem. Exactly the same procedure can be performed for the problem $\left(\mathrm{P}_{4}\right)$ with only minor changes so that we obtain the following theorem.

Theorem 4.1. There exists at least one solution to the problem $\left(P_{4}\right)$. Moreover, the following maximum principle holds.

Theorem 4.2. If $W \geq 0$ on $\Gamma_{I} \times[0, T]$ and $\theta_{0} \geq 0$; then $p \geq 0$ a.e. in $\Omega \times[0, T]$

Proof. Following [19], we build the sequence $\left(p_{\varepsilon}\right)$ of solution of the problems

$$
\begin{aligned}
-\varepsilon \frac{\partial p_{\varepsilon}}{\partial t}+p_{\varepsilon} & =p^{-} \text {on }[0, T] \\
p_{\varepsilon}(T) & =0
\end{aligned}
$$

in the Banach space $H^{1}(\Omega)$. From the classical Cauchy-Lipshitz-Picard theorem [12: p. $104]$, there exists a unique solution $p_{\varepsilon} \in C^{1}\left([0, T] ; H^{1}(\Omega)\right)$ of problem (4.18) - (4.19). Multiplying (4.18) by $p_{e}$ and $\varepsilon \frac{\partial p_{c}}{\partial t}$ and integrating over $Q$ we obtain

$$
\left\|p_{e}\right\|_{L^{2}(Q)} \leq\left\|p^{-}\right\|_{L^{2}(Q)}
$$


and

$$
\left\|\varepsilon \frac{\partial p_{\varepsilon}}{\partial t}\right\|_{L^{2}(Q) \|} \leq\left\|p^{-}\right\|_{L^{2}(Q)}
$$

respectively. Differentiating (4.18) with respect to $x$, multiplying by $\nabla p_{e}$ and then integrating over $Q$ we get

$$
\left\|\nabla p_{e}\right\|_{L^{2}(Q)} \leq\left\|\nabla p^{-}\right\|_{L^{2}(Q)}
$$

From $(4.20)-(4.22)$ we deduce that there exist $\tilde{p} \in L^{2}\left(0, T ; H^{1}(\Omega)\right.$ and $\zeta \in L^{2}(\mathbb{Q})$ such that

$$
\begin{aligned}
p_{\varepsilon} & \longrightarrow \tilde{p} \quad \text { weakly in } L^{2}\left(0, T ; H^{1}(\Omega)\right) \\
\varepsilon \frac{\partial p_{\varepsilon}}{\partial t} & \longrightarrow \zeta \quad \text { weakly in } L^{2}\left(0, T ; H^{1}(\Omega)\right)
\end{aligned}
$$

From (4.23), $\varepsilon p_{\varepsilon} \rightarrow 0$ in $L^{2}\left(0, T ; H^{1}(\Omega)\right)$, therefore $\zeta=0$. Passing to the limit in (4.18), we deduce that $\tilde{p}=p^{-}$a.e. in $Q$. Taking now $p_{\varepsilon}$ as a test function in (4.16) and passing to the limit over $\varepsilon$, we deduce

$$
-\frac{1}{12 \nu} \int_{Q} h^{3}\left|\nabla p^{-}\right|^{2} \geq \int_{\Sigma} W p^{-}
$$

as $W \geq 0$, therefore $p^{-}=0$ a.e. in $Q$, i.e. $p \geq 0$ a.e. in $Q$.

\section{About the uniqueness of problem $\left(P_{4}\right)$}

5.1 Partial results of uniqueness. Partial results of uniqueness appeared in [13]. They are valid; however, only when the solution is a limit solution of parabolic regularized formulation.

Using a similar Hele-Shaw problem approach, we will prove a uniqueness result for problem $\left(\mathrm{P}_{4}\right)$ in the particular case where $V=0$, with no conditions, neither about the sign of $\frac{\partial h}{\partial t}$, nor on the regularity of the free boundary. For this, we first give an other equivalent weak formulation of the problem $\left(\mathrm{P}_{4}\right)$. Then we will introduce in Theorem 5.1 a second variational approach.

5.2 An other equivalent weak formulation of the problem $\left(P_{3}\right)$. The purpose of this subsection is the study of a new formulation of the problem $\left(\mathrm{P}_{3}\right)$ which enables us, when shear effects are cancelled to obtain a uniqueness result.

Remark 5.1. As $H_{0}^{1}(0, T ; \mathbb{W})$ is dense in $L^{2}(0, T ; \mathbb{W})$, with $\mathbb{W}=\left\{\varphi \in H^{1}(\Omega)\right.$ : $\varphi=0$ on $\left.\Gamma_{e x}\right\}$, we can write $(4.16)$ in the form

$$
\int_{0}^{T}\left\langle\frac{\partial \theta h}{\partial t}, \varphi\right\rangle+\frac{1}{12 \nu} \int_{Q} h^{3} \nabla p \nabla \varphi-\int_{Q} \theta h V \nabla \varphi=\int_{\Sigma_{1}} W \varphi \quad(\varphi \in W)
$$


The Transient Lubrication Problem

75

a.e. in $[0, T]$.

Remark 5.2. Using Remark 5.1 , the problem $\left(\mathrm{P}_{4}\right)$ is equivalent to the following problem

$\left(\mathbf{P}_{5}\right)$ Find a pair $(p, \theta) \in L^{2}(0, T ; \mathbb{W}) \times\left(L^{\infty}(\mathbb{Q}) \cap H^{1}\left(0, T ; H^{-1}(\Omega)\right)\right)$ satisfying, for all $\varphi \in \mathbb{W}$

$$
\begin{array}{rlrl}
0 \leq \theta \leq 1 & \text { and } p(1-\theta)=0 & \text { ace. in } \Omega \times[0, T] \\
\left\langle\frac{\partial \theta h}{\partial t}, \varphi\right\rangle+\frac{1}{12 \nu} \int_{\Omega} h^{3} \nabla p \nabla \varphi-\int_{\Omega} \theta h V \nabla \varphi & =\int_{\Gamma_{\mathbf{I}}} W \varphi & & \text { ace. in }[0, T] \\
\left.\theta \quad \theta\right|_{t=0} & =\theta_{0} . & & \text { in } H^{-1}(\Omega) .
\end{array}
$$

Theorem 5.1. Let $W \geq 0$ and $h=l(t) g(x)$. If $(p, \theta)$ is a solution of problem $\left(\mathrm{P}_{5}\right)$ with $V=0$, then $z$ (given by the definition (3.8)) is a solution of the following variational problem:

$\left(\mathbf{P}_{6}\right)$ Find $z \in K$ such that, for all $\psi \in K$,

$$
\begin{aligned}
\int_{\Omega} g^{3} \nabla z \nabla\left(\psi-\frac{\partial z}{\partial t}\right) & \geq \int_{\Omega}\left(\theta_{0} h_{0}-h\right)\left(\psi-\frac{\partial z}{\partial t}\right)+\int_{\Gamma_{1}} Q\left(\psi-\frac{\partial z}{\partial t}\right) \\
z(0) & =0
\end{aligned}
$$

where $Q$ is given by the definition (3.8).

Proof. As $V=0$, equation (5.2) reduces to.

$$
\left\langle\frac{\partial \theta h}{\partial t}, \varphi\right\rangle+\frac{1}{12 \nu} \int_{\Omega} h^{3} \nabla p \nabla \varphi=\int_{\Gamma_{1}} W \varphi \quad \text { ace. in }[0, T]
$$

for all $\varphi \in \mathbb{W}$. Then by time integration

$$
\left\langle\int_{0}^{t} \frac{\partial \theta h}{\partial t}, \varphi\right\rangle+\int_{0}^{t} \int_{\Omega} \frac{h^{3}}{12 \nu} \nabla p \nabla \varphi=\iint_{0}^{t} \int_{\Gamma_{I}} W \dot{\varphi}
$$

for all $\phi \in \mathbb{V}$ as $h=l(t) g(x)$ and, using (3.8), we obtain

$$
\left\langle\theta h-\theta_{0} h_{0}, \varphi\right\rangle+\int_{\Omega} g^{3} \nabla z \nabla \varphi=\int_{\Gamma_{1}} Q \varphi
$$

for all $\dot{\phi} \in \mathbb{W}$. Putting now $\varphi=\psi-\frac{\partial z}{\partial t}$ for all $\psi \in \mathbb{W}$ yields

$$
\begin{aligned}
\int_{\Omega} g^{3} \nabla z \nabla\left(\psi-\frac{\partial z}{\partial t}\right)= & \int_{\Gamma_{1}} Q\left(\psi-\frac{\partial z}{\partial t}\right)+\int_{\Omega}\left(\theta_{0} h_{0}-h\right)\left(\psi-\frac{\partial z}{\partial t}\right) \\
& +\int_{\Omega}(1-\theta) h\left(\psi-\frac{\partial z}{\partial t}\right)
\end{aligned}
$$


and as

$$
\int_{\Omega}(1-\theta) h\left(\psi-\frac{\partial z}{\partial t}\right)=\int_{\Omega}(1-\theta) h \psi \quad(0 \leq \psi \in \mathbb{W})
$$

we deduce the required inequality

$$
\int_{\Omega} g^{3} \nabla z \nabla\left(\psi-\frac{\partial z}{\partial t}\right) \geq \int_{\Omega}\left(\theta_{0} h_{0}-h\right)\left(\psi-\frac{\partial z}{\partial t}\right)+\int_{\Gamma_{\mathbf{I}}} Q\left(\psi-\frac{\partial z}{\partial t}\right)
$$

for all $\psi \in K$. Moreover, the definition of $z$ (see (3.8)) gives $z(0)=0$.

In the following subsection, we will prove the uniqueness of the solution of such problem by studying a more general formulation.

5.3 A second abstract variational approach. Let $f=f_{1}+f_{2}$ with $f_{1} \in L^{\infty}(\Omega)$ and $f_{2} \in C^{1}(\bar{\Omega} \times[0, T])$ and $G \in C^{1}([0, T] \times \bar{\Omega})$ with $\frac{\partial G}{\partial t} \geq 0$. Consider the following problem

(PZ) Find $z \in C^{0}(0, T ; \mathbb{W})$ such that $\frac{\partial z}{\partial t} \in L^{2}(0, T ; \mathbb{W}) \cap K$ and, for all $\varphi \in \mathbb{W}$,

$$
\begin{aligned}
a\left(z, \varphi-\frac{\partial z}{\partial t}\right)+I_{K}(\varphi)-I_{K}\left(\frac{\partial z}{\partial t}\right) & \geq\left(f, \varphi-\frac{\partial z}{\partial t}\right)+\left\langle G, \varphi-\frac{\partial z}{\partial t}\right\rangle \\
z(0) & =0
\end{aligned}
$$

where

$$
I_{K}=\left\{\begin{array}{ll}
0 & \text { for } \varphi \in K \\
+\infty & \text { for } \varphi \notin K
\end{array} \quad \text { for } \quad K=\{\varphi \in \mathbb{W}: \varphi \geq 0\}\right.
$$

$a(\cdot, \cdot)$ is the bilinear continuous symmetric and coercive form on $H^{1}(\Omega)$ with the associated second order operator $A$ defined for problem $\left(\mathrm{P}_{2}\right),(\cdot, \cdot)$ denotes the usual inner product in $L^{2}(\Omega)$ and $\langle\cdot, \cdot\rangle$ defines the duality in $L^{2}\left(\Gamma_{\mathrm{I}}\right)$. .

To prove the existence and uniqueness of the solution of problem (PZ), we hope to apply in the space Proposition II.9 of [11]. The linear form defined by $\psi \rightarrow$ $(f(t), \psi)+\langle G(t), \psi\rangle$ from $\mathbb{W}$ to $\mathbb{R}$ is continuous. Using the density of $\mathbb{W}$ in $L^{2}(\Omega)$ and the Riesz theorem, there exists a unique function $F \in L^{2}(\Omega)$ such that

$$
(f(t), \psi)+\langle G(t), \psi\rangle=(F(t), \psi) \quad \text { for all } \psi \in \mathbb{W} .
$$

But the required assumption $F \in \mathbb{V}$ is not satisfied as in our initial problem $f_{1}=\chi_{0} h_{0}$ and this is a characteristic of our problem. To cope with, we regularize $f_{1}$ using the convolution between $f_{1}$ and the mollifiers functions $\xi_{\varepsilon}$ (see, for example, [12: p. 66]). Let $f_{1}^{\varepsilon}=\xi_{\varepsilon} * f_{1}$ so that $f_{1}^{\varepsilon}$ lies in $H^{1}(\Omega) \cap L^{\infty}(\Omega)$ and $f_{1}^{\varepsilon} \rightarrow f_{1}$ as $\varepsilon \rightarrow 0$. We define $f_{\varepsilon}$ by $f_{\varepsilon}=f_{1}^{\epsilon}+f_{2}$ and $F_{\varepsilon}$ by

$$
\left(F_{\varepsilon}(t), \psi\right)=\left(f_{\varepsilon}(t), \psi\right)+(G(t), \psi) \quad(\psi \in \mathbb{W}) .
$$

Let us consider first the following approximation problem: 
$\left(\mathbf{P Z}_{\varepsilon}\right)$ For $\varepsilon \in(0,1)$ find $z_{\varepsilon} \in C^{0}(0, T ; \mathbb{W})$ with $\frac{\partial z_{\varepsilon}}{\partial \varepsilon} \in L^{2}(0, T ; \mathbb{V}) \cap K$ such that, for all $\varphi \in K$,

$$
\begin{aligned}
a\left(z_{\varepsilon}, \varphi-\frac{\partial z_{\varepsilon}}{\partial t}\right) & \geq\left(F_{\epsilon}, \varphi-\frac{\partial z_{\varepsilon}}{\partial t}\right) \quad \text { a.e. in }(0, T) \\
z_{\varepsilon}(0) & =0
\end{aligned}
$$

Here $F_{\varepsilon} \in C^{0}\left(0, T ; H^{1}(\Omega)\right)$ and $\frac{\partial F_{\varepsilon}}{\partial t} \in L^{2}\left(0, T ; H^{1}(\Omega)\right)$. Then from a theorem of Kato, appearing in [11: p. 80], there exists a unique solution $z_{\varepsilon} \in C^{0}\left(0, T ; H^{1}(\Omega)\right)$ of problem $\left(\mathrm{PZ} Z_{\varepsilon}\right)$, such that $z_{\varepsilon}$ has right derivative in all $t \in[0, T]$ and

$$
\left\|\frac{\partial z_{\varepsilon}}{\partial t}(t)\right\|_{H^{1}(\Omega)} \leq \int_{0}^{t}\left\|\frac{\partial F_{\varepsilon}}{\partial t}(\dot{s})\right\|_{H^{1}(\Omega)} d s .
$$

However, $f_{1}$ and $f_{1}^{\ell}$ are not functions of the time, so $\frac{\partial F_{l}}{\partial t}(s)=\frac{\partial F}{\partial t}(s)$. Therefore

$$
\left\|\frac{\partial z_{\varepsilon}}{\partial t}(t)\right\|_{H^{1}(\Omega)} \leq \int_{0}^{t}\left\|\frac{\partial F}{\partial t}(s)\right\|_{H^{1}(\Omega)} d s
$$

and from [11: Proposition II.9]

$$
\frac{\partial z_{\varepsilon}}{\partial t} \in L^{\infty}\left(\delta, T ; H^{1}(\Omega)\right) \quad \text { for all } \delta \in(0, T)
$$

Thus the statement is proved.

The following propositions give some a priori estimates for $z_{\varepsilon}(t)$.

Proposition 5.1. If $z_{\varepsilon}$ is the unique solution of problem $\left(\mathrm{PZ}_{\varepsilon}\right)$, then the estimate

$$
\left\|z_{e}(t)\right\|_{H^{1}(\Omega)} \leq t \int_{0}^{T}\left\|\frac{\partial F}{\partial t}(s)\right\|_{H^{i}(\Omega)} d s
$$

is true.

Proof. We have

$$
\nabla z_{e}(t)=\int_{0}^{t} \nabla\left(\frac{\partial z_{e}}{\partial t}(s)\right) d s \quad \text { where } \nabla=\left(\frac{\partial}{\partial x_{1}}, \frac{\partial}{\partial x_{2}}\right)^{\top}
$$

Using the Cauchy-Schwarz inequality we obtain

$$
\left|\nabla z_{e}(t)\right|^{2}=\left(\int_{0}^{t} \nabla\left(\frac{\partial z_{\varepsilon}}{\partial t}(s)\right) d s\right)^{2} \leq t\left(\int_{0}^{t}\left|\nabla\left(\frac{\partial z_{\varepsilon}}{\partial t}(s)\right)\right|^{2} d s\right)
$$


so

$$
\int_{\Omega}\left|\nabla z_{\epsilon}(t)\right|^{2} d t \leq t \int_{0}^{t}\left\|\frac{\partial z_{e}}{\partial t}(s)\right\|_{H^{1}(\Omega)}^{2} d s .
$$

Using (5.5) we deduce

$$
\begin{aligned}
\left\|z_{\varepsilon}(t)\right\|_{H^{1}(\Omega)}^{2} & \leq t \int_{0}^{t}\left(\int_{0}^{s}\left\|\frac{\partial F}{\partial t}(\tau)\right\|_{H^{1}(\Omega)} d \tau\right)^{2} d s \\
& \leq t\left(\int_{0}^{t} d s\right)\left(\int_{0}^{T}\left\|\frac{\partial F}{\partial t}(\tau)\right\|_{H^{1}(\Omega)} d \tau\right)^{2}
\end{aligned}
$$

thus

$$
\left\|z_{\varepsilon}(t)\right\|_{H^{1}(\Omega)}^{2} \leq t^{2}\left(\int_{0}^{T}\left\|\frac{\partial F}{\partial t}(\tau)\right\|_{H^{1}(\Omega)} d \tau\right)^{2}
$$

Taking the square root of the two members of this inequality we get the result.

Proposition 5.2. If $z_{\varepsilon}$ is the unique solution of the problem $\left(\mathrm{PZ}_{\varepsilon}\right)$, then the inclusion

$$
z_{\varepsilon}(t) \in H^{2}(\Omega)
$$

is true.

Proof. Following [11: p. 120] we consider the problem

$$
\begin{aligned}
z_{\eta}(t)+\eta A z_{\eta}(t) & =z_{\varepsilon}(t) & & \text { in } \Omega \\
z_{\eta}(t) & =0 & & \text { on } \Gamma_{\mathbf{e x}} \\
\sum_{i, j=1}^{2} a_{i j} \frac{\partial z_{\eta}}{\partial x_{i}}(t) \cos \left(n, x_{j}\right) & =G(t) & & \text { on } \Gamma_{\mathrm{I}} \\
z_{\eta}(0) & =0 & &
\end{aligned}
$$

where $z_{\varepsilon}$ is the unique solution of problem $(\mathrm{PZ} \varepsilon)$. As $\frac{\partial z_{\varepsilon}}{\partial t} \in L^{2}\left(0, T ; H^{1}(\Omega)\right)$ we have $\frac{\partial z_{\eta}}{\partial t} \in L^{2}\left(0, T ; H^{2}(\Omega)\right)$ and

$$
\begin{array}{rlrl}
\frac{\partial z_{\eta}}{\partial t}+\eta A\left(\frac{\partial z_{\eta}}{\partial t}\right) & =\frac{\partial z_{\eta}}{\partial t} \quad \text { in } \Omega, \text { a.e. in }(0, T) \\
\frac{\partial z_{\eta}}{\partial t} & =0 & \text { on } \Gamma_{\mathrm{ex}} \\
\sum_{i, j=1}^{2} a_{i j} \frac{\partial}{\partial x_{i}}\left(\frac{\partial z_{\eta}}{\partial t}\right) \cos \left(n, x_{j}\right) & =\frac{\partial G}{\partial t} & & \text { on } \Gamma_{\mathrm{l}} .
\end{array}
$$


Since $\frac{\partial G}{\partial t} \geq 0$ on $\Gamma_{1}$ and $\frac{\partial x_{t}}{\partial t} \geq 0$ in $\Omega$, a.e. in $(0, T)$, then by the maximum principle we have $\frac{\partial z_{\eta}}{\partial t} \geq 0$ in $\Omega$, a.e in $(0, T)$. Consequently, we can use $\frac{\partial z_{\eta}}{\partial t}$ as a test function in problem $(\mathrm{PZ} \varepsilon)$, so

$$
a\left(z_{\varepsilon}(s), \frac{\partial z_{\eta}}{\partial t}(s)-\frac{\partial z_{\varepsilon}}{\partial t}(s)\right) \geq\left(F_{e}(s), \frac{\partial z_{\eta}}{\partial t}(s)-\frac{\partial z_{\varepsilon}}{\partial t}(s)\right)
$$

as

$$
\begin{aligned}
& \frac{1}{2} a\left(z_{\eta}(t)-z_{\varepsilon}(t), z_{\eta}(t)-z_{\varepsilon}(t)\right) \\
& \quad=\int_{0}^{t} a\left(z_{\eta}(s)-z_{\varepsilon}(s), \frac{\partial z_{\eta}}{\partial t}(s)-\frac{\partial z_{\varepsilon}}{\partial t}(s)\right) d s \\
& \quad=\int_{0}^{t} a\left(z_{\eta}(s), \frac{\partial z_{\eta}}{\partial t}(s)-\frac{\partial z_{\varepsilon}}{\partial t}(s)\right) d s-\int_{0}^{t} a\left(z_{\varepsilon}(s), \frac{\partial z_{\eta}}{\partial t}(s)-\frac{\partial z_{\varepsilon}}{\partial t}(s)\right) d s .
\end{aligned}
$$

Then taking (5.11) in (5.12), we get

$$
\begin{aligned}
& \frac{1}{2} a\left(z_{\eta}(t)-z_{\varepsilon}(t), z_{\eta}(t)-z_{\varepsilon}(t)\right) \\
& \quad \leq \int_{0}^{t} a\left(z_{\eta}(s), \frac{\partial z_{\eta}}{\partial t}(s)-\frac{\partial z_{\varepsilon}}{\partial t}(s)\right) d s-\int_{0}^{t}\left(F_{\varepsilon}(s), \frac{\partial z_{\eta}}{\partial t}(s)-\frac{\partial z_{\ell}}{\partial t}(s)\right) d s .
\end{aligned}
$$

Using the Green formula we have

$$
\begin{aligned}
& \int_{0}^{t} \int_{\Omega} A z_{\eta}(s)\left(\frac{\partial z_{\eta}}{\partial t}(s)-\frac{\partial z_{\varepsilon}}{\partial t}(s)\right) d x d s \\
& \quad-\int_{0}^{t} \int_{\Omega} F_{\varepsilon}(s)\left(\frac{\partial z_{\eta}}{\partial t}(s)-\frac{\partial z_{\varepsilon}}{\partial t}(s)\right) d x d s+\int_{0}^{t} \int_{\Gamma_{1}} G\left(\frac{\partial z_{\eta}}{\partial t}(s)-\frac{\partial z_{\varepsilon}}{\partial t}(s)\right) d \tau d s \\
& \quad \geq \frac{1}{2} a\left(z_{\eta}(t)-z_{\varepsilon}(t), z_{\eta}(t)-z_{\varepsilon}(t)\right)
\end{aligned}
$$

and from $(5.4)_{b}$, this inequality becomes

$$
\begin{gathered}
\int_{0}^{t} \int_{\Omega} A z_{\eta}(s)\left(\frac{\partial z_{\eta}}{\partial t}(s)-\frac{\partial z_{\varepsilon}}{\partial t}(s)\right) d x d s-\int_{0}^{t} \int_{\Omega} f_{\varepsilon}(s)\left(\frac{\partial z_{\eta}}{\partial t}(s)-\frac{\partial z_{e}}{\partial t}(s)\right) d x d s \\
\geq \frac{1}{2} a\left(z_{\eta}(t)-z_{\varepsilon}(t), z_{\eta}(t)-z_{\varepsilon}(t)\right) \geq 0
\end{gathered}
$$

whence

$$
\int_{0}^{t} \int_{\Omega}\left(A z_{\eta}(s)-f_{e}(s)\right)\left(\frac{\partial z_{\eta}}{\partial t}(s)-\frac{\partial z_{\epsilon}}{\partial t}(s)\right) d x d s \geq 0
$$


Putting now

$$
\Phi_{\eta, \varepsilon}:=-A z_{\eta}+f_{\varepsilon}
$$

and using (5.10) we have

$$
\frac{\partial z_{\eta}}{\partial t}-\frac{\partial z_{\varepsilon}}{\partial t}=\eta\left(\frac{\partial \Phi_{\eta, e}}{\partial t}-\frac{\partial f_{\varepsilon}}{\partial t}\right)
$$

From (5.13) - (5.15) we get

$$
\int_{0}^{t} \int_{\Omega} \Phi_{\eta, \varepsilon}\left(\frac{\partial \Phi_{\eta, \varepsilon}}{\partial t}-\frac{\partial f_{\varepsilon}}{\partial t}\right) d x d s \leq 0
$$

Therefore

$$
\begin{aligned}
\int_{0}^{t} \int_{\Omega} \Phi_{\eta, \varepsilon} \frac{\partial f_{\varepsilon}}{\partial t} d x d s & \geq \int_{0}^{t} \int_{\Omega} \Phi_{\eta, \varepsilon} \frac{\partial \Phi_{\eta, \varepsilon}}{\partial t} d x d s \\
& =\frac{1}{2} \int_{\Omega}\left|\Phi_{\eta, \varepsilon}(t)\right|^{2} d x-\frac{1}{2} \int_{\Omega}\left|\Phi_{\eta, \varepsilon}(0)\right|^{2} d x
\end{aligned}
$$

so

$$
\begin{aligned}
\frac{1}{2}\left\|\Phi_{\eta, e}(t)\right\|_{L^{2}(\Omega)}^{2} \leq & \frac{1}{2}\left\|\Phi_{\eta, e}(0)\right\|_{L^{2}(\Omega)}^{2} \\
& +\int_{0}^{t}\left\|\Phi_{\eta, e}(s)\right\|_{L^{2}(\Omega)}\left\|\frac{\partial f_{\varepsilon}(s)}{\partial t}(s)\right\|_{L^{2}(\Omega)} d s .
\end{aligned}
$$

Remember the following Gronwall inequality (see [9: p. 15]):

Let $I \subset \mathbb{R}^{+}, \varphi \in C\left(I ; \mathbb{R}^{+}\right)$and $\psi \in L_{\mathrm{loc}}^{1}\left(I ; \mathbb{R}^{+}\right)$such that

$$
\frac{1}{2} \varphi(t)^{2} \leq \frac{1}{2} \varphi(s)^{2}+\int_{s}^{t} \psi(\tau) \varphi(\tau) d \tau \quad \text { for all } \quad s, t \in I, s \leq t
$$

Then

$$
\varphi(t) \leq \varphi(s)+\int_{s}^{t} \psi(\dot{\tau}) d \tau \quad \text { for all } s, t \in I, s \leq t
$$

Using this inequality, the previous one gives

$$
\left\|\Phi_{\eta, \varepsilon}(t)\right\|_{L^{2}(\Omega)} \leq\left\|\Phi_{\eta, \varepsilon}(0)\right\|_{L^{2}(\Omega)}+\int_{0}^{t}\left\|\frac{\partial f_{\varepsilon}}{\partial s}(s)\right\|_{L^{2}(\Omega)} d s \quad(t \in(0, T)) .
$$


As $f_{e}(s)=f_{1}^{e}+f_{2}(s)$ with $f_{1}^{\epsilon} \in H^{1}(\Omega)$ and $f_{2} \in C^{1}(\bar{\Omega} \times(0, T))$ and $z_{\eta}(0)=0$, then

$$
\left\|\Phi_{\eta, \varepsilon}(t)\right\|_{L^{2}(\Omega)} \leq\left\|f_{\varepsilon}(0)\right\|_{i^{2}(\Omega)}+\int_{0}^{t}\left\|\frac{\partial f_{2}}{\partial s}(s)\right\|_{L^{2}(\Omega)} d s \quad(t \in(0, T)) .
$$

So from (5.14) we get

$$
\left\|-A z_{\eta}+f_{e}\right\|_{L^{2}(\Omega)} \leq\left\|f_{e}(0)\right\|_{L^{2}(\Omega)}+\int_{0}^{t}\left\|\frac{\partial f_{2}}{\partial s}(s)\right\|_{L^{2}(\Omega)} d s \quad(t \in(0, T)) .
$$

Then $\left\|z_{\eta}\right\|_{H^{2}(\Omega)} \leq C_{1}\left\|A z_{\eta}\right\|_{L^{2}(\Omega)} \leq C_{2}$, where $C_{1}$ and $C_{2}$ are constants independent of $\eta$. Then $z_{\eta}(t)$ weakly converges in $H^{2}(\Omega)$. Moreover, from (5.9) and (5.14) we can write

$$
z_{\eta}(t)-z_{e}(t)=\eta \Phi_{\eta, e}(t)-\eta f_{e}(t)
$$

Then

$$
\lim _{\eta \rightarrow 0}\left\|z_{\eta}(t)-z_{e}(t)\right\|_{L^{2}(\Omega)} \leq \lim _{\eta \rightarrow 0} \eta\left\|\Phi_{\eta, e}(t)\right\|_{L^{2}(\Omega)}=0 .
$$

So the $H^{2}(\Omega)$ weak limit of $z_{\eta}(t)$ is exactly $z_{e}(t)$ which completes the proof.

Theorem 5.2. There exists a unique solution $z$ of problem (PZ).

Proof. From problem $\left(\mathrm{PZ}_{\varepsilon}\right)$ we have

$$
a\left(z_{\varepsilon}, \varphi-\frac{\partial z_{\varepsilon}}{\partial t}\right) \geq\left(F_{\varepsilon}, \varphi-\frac{\partial z_{\varepsilon}}{\partial t}\right) \quad \text { for all } \varphi \in K, \text { a.e. in }(0, T)
$$

which is equivalent to

$$
\begin{aligned}
& a\left(z_{\varepsilon}, \frac{\partial z_{e}}{\partial t}\right)=\left(F_{e}, \frac{\partial z_{e}}{\partial t}\right) \\
& a\left(z_{e}, \varphi\right) \geq\left(F_{e}, \varphi\right) \text { for all } \varphi \in K, \text { a.e. in }(0, T)
\end{aligned}
$$

From (5.7) there exists $z(t) \in L^{2}\left(0, T ; H^{1}(\Omega)\right)$ such that $z_{e}(t)$ tends towards $z(t)$ with

$$
\dot{a}(z, \varphi) \geq(\dot{F}, \varphi)
$$

Moreover, from (5.5) we deduce that, for all $\varphi \in D\left(0, T ; L^{2}(\Omega)\right)$,

$$
\lim _{e \rightarrow 0}\left\langle\frac{\partial z_{\varepsilon}}{\partial t}, \dot{\varphi}\right\rangle=-\lim _{e \rightarrow 0}\left\langle z_{e}, \frac{\partial \varphi}{\partial t}\right\rangle=-\left\langle z, \frac{\partial \varphi}{\partial t}\right\rangle \doteq\left\langle\frac{\partial \varphi}{\partial t}, \varphi\right\rangle .
$$

In the previous line $\langle\cdot, \cdot\rangle$ denotes the duality between the spaces $\underset{:}{D}\left(0, T ; L^{2}(\Omega)\right)$ and $D^{\prime}\left(0, T ; L^{2}(\Omega)\right)$, whence

$$
\lim _{\varepsilon \rightarrow 0}\left\langle\frac{\partial z_{\varepsilon}}{\partial t}, \varphi\right\rangle=\left\langle\frac{\partial z}{\partial t}, \phi\right\rangle, \text { for all } \varphi \in D\left(0, T ; L^{2}(\Omega)\right)
$$


And from (5.18) and $(5.4)_{b}$ we have

$$
a\left(z_{\ell}, \frac{\partial z_{\varepsilon}}{\partial t}\right)-\left\langle G, \frac{\partial z_{\varepsilon}}{\partial t}\right\rangle-\left(f_{\varepsilon}, \frac{\partial z_{\varepsilon}}{\partial t}\right)=0
$$

Then

$$
\left(-A z_{e}+f_{\varepsilon}, \frac{\partial z_{e}}{\partial t}\right)=0
$$

As

$$
\lim _{\varepsilon \rightarrow 0}\left\langle-A z_{\varepsilon}+f_{\varepsilon}, \psi\right\rangle=\lim _{\varepsilon \rightarrow 0} a\left(z_{\varepsilon}, \psi\right)+(f, \psi) \quad\left(\psi \in D^{\prime}(\Omega)\right)
$$

from (5.7) there exist $z(t) \in L^{2}\left(0, T ; H^{1}(\Omega)\right)$ such that, for all $\psi \in D^{\prime}(\Omega)$,

$$
\lim _{\varepsilon \rightarrow 0}\left\langle-A z_{\varepsilon}+f_{e}, \psi\right\rangle=a(z, \psi)+(f, \psi)=\langle-A z+f, \psi\rangle
$$

From (5.21) and (5.5), $\frac{\partial z_{t}}{\partial t}$ tends strongly towards $\frac{\partial z}{\partial t}$ in $L^{2}(\Omega)$, thus (5.23) and (5.22) gives $\left(-A z+f, \frac{\partial z}{\partial t}\right)=0$. Then by the Green formula we get

$$
a\left(z, \frac{\partial z}{\partial t}\right)=\left(f, \frac{\partial z}{\partial t}\right)+\left\langle G, \frac{\partial z}{\partial t}\right\rangle
$$

Thus by $(5.4)_{a}$ yields

$$
a\left(z(t), \frac{\partial z}{\partial t}(t)\right)=\left(F(t), \frac{\partial z}{\partial t}(t)\right) .
$$

From (5.20) and (5.24) we deduce that $z$ satisfies the variational inequality

$$
a\left(z(t), \varphi-\frac{\partial z}{\partial t}(t)\right) \geq\left(F(t), \varphi-\frac{\partial z}{\partial t}(t)\right) \quad(\varphi \in \mathbb{W})
$$

with

$$
z(0)=0 \text {. }
$$

Using now [11: Proposition II.9]. As $F \in L^{2}\left(0, T ; L^{2}(\Omega)\right)$ there exists one and only one $z \in C^{0}\left(0, T ; L^{2}(\Omega)\right)$ which is solution of the variational problem (5.25) - (5.26), such that $\frac{\partial z}{\partial t} \in L^{2}(0, T ; W)$. Consequently, as $C^{0}(0, T ; \mathbb{V}) \subset C^{0}\left(0, T ; L^{2}(\Omega)\right)$, we have both existence and uniqueness for $z \in C^{0}(0, T ; W)$ as solution of problem (5.25) - (5.26), such that $\frac{\partial z}{\partial t} \in L^{2}(0, T ; W)$. And by $(5.4)_{a}$ the problem (5.25) - (5.26) is the variational problem (PZ).

5.4 Application to the particular problem (P6). The results of the last subsection enables us to conclude about the existence of solution for problem $\left(P_{6}\right)$, and then for problem $\left(\mathrm{P}_{4}\right)-\left(\mathrm{P}_{5}\right)$ when $V=0$, which is nothing else than a particular case of problem (PZ).

Theorem 5.3. Let $W \geq 0$ and $h=l(t) g(x)$. Then there exists a unique $z \in$ $C^{0}(0, T ; \mathbb{W})$ with $\frac{\partial z}{\partial t} \in L^{2}(0, T ; \mathbb{W} \cap K)$, which is solution of the problem $\left(\mathrm{P}_{6}\right)$.

Proof. Choosing $f_{1}=\theta_{0} h_{0}, f_{2}=-h, G=Q$ and $a(z, \varphi)=\int_{\Omega} g^{3} \nabla z \nabla \varphi$ in problem $(\mathrm{PZ})$, the result follows immediately from the previous subsection. 
Theorem 5.4. Let $W \geq 0$ and $h=l(t) g(x)$. Then there exists a unique solution $(p, \theta)$ of problem $\left(\mathrm{P}_{4}\right)-\left(\mathrm{P}_{5}\right)$ with $V=0$.

Proof. From Theorem 4.1, 5.1 and 5.3 we have existence and uniqueness result for $p$. Moreover, if $\left(p, \theta_{1}\right)$ and $\left(p, \theta_{2}\right)$ are two solutions of problem $\left(\mathrm{P}_{4}\right)-\left(\mathrm{P}_{5}\right)$ with $V=0$, then from (5.2) we have $\left.\left\langle\frac{\partial}{\partial t}\left(\theta_{1}-\theta_{2}\right) h\right), \varphi\right\rangle=0$ a.e in $[0, T]$. By time integration between 0 and $t \in(0, T]$ and using (5.4), this implies $\left\langle\left(\theta_{1}(t)-\theta_{2}(t)\right) h(t), \varphi\right\rangle=0$ so that $\theta_{1}=\theta_{2}$ in $\mathbb{W}^{\prime}$.

\section{Connection between the two variational approachs $\left(\mathrm{P}_{2}\right)$ and $(\mathrm{PZ})$}

The next section allows us to prove that the two variational formulations introduced in the previous sections are indeed related with the same solution.

Theorem 6.1. If $z$ is the solution of problem $\left(\mathrm{P}_{2}\right)$, then $z$ satisfies the inequality

$$
a\left(\frac{\partial z}{\partial t}, v-z(t)\right) \leq\left(\frac{\partial F}{\partial t}, v-z(t)\right)+\langle G, v-z(t)\rangle
$$

for all $v \in K$ and is also the solution of the variational inequality with constraint on $\frac{\partial z}{\partial t}$

$$
a\left(z, \varphi-\frac{\partial z}{\partial t}\right)+I_{k}(\varphi)-I_{k}\left(\frac{\partial z}{\partial t}\right) \geq\left(F, \varphi-\frac{\partial z}{\partial t}\right)+\left\langle G, \varphi-\frac{\partial z}{\partial t}\right\rangle
$$

for all $\varphi_{1} \in \mathbb{W}$ where $I_{k}(\varphi)= \begin{cases}0 & \text { if } \varphi \in K \\ \infty & \text { if } \varphi \notin K\end{cases}$

Proof. To show inequality (6.1) we will first establish the inequality

$$
\begin{aligned}
& a(z(t)-z(s), \varphi-z(s)) \\
& \quad \leq(F(t)-F(s), \varphi-z(s))+\langle G(t)-G(s), \varphi-z(s)\rangle
\end{aligned}
$$

for all $\varphi \in K$ and for $0 \leq s \leq t<T$. To do that, we choose $\varphi-z_{e}(s)$, where $z_{e}$ is the unique solution of problem $\left(\mathrm{PZ}_{e}\right)$, as test function in (3.16). Then

$$
\begin{aligned}
& a\left(z_{e}(t)-z_{e}(s), \varphi-z_{\varepsilon}(s)\right)+\left(e(t) H_{\varepsilon}\left(z_{e}(t)\right)-e(s) H_{e}\left(z_{e}(s)\right), \varphi-z_{e}(s)\right) \\
& =\left(F(t)+e(t)-F(s)-e(s), \varphi-z_{e}(s)\right)+\left\langle G(t)-G(s), \varphi-z_{e}(s)\right\rangle
\end{aligned}
$$

which can be rewritten as

$$
\begin{aligned}
a\left(z_{e}(t)\right. & \left.-z_{e}(s), \varphi-z_{e}(s)\right) \\
= & \left(\left(F(t)-F(s), \varphi-z_{\varepsilon}(s)\right)+\left\langle G(t)-G(s), \varphi-z_{e}(s)\right\rangle\right) \\
& +\left((e(s)-e(t))\left(H_{e}\left(z_{e}(s)\right)-1\right), \varphi-z_{e}(s)\right) \\
& +\left(-e(t)\left(H_{e}\left(z_{\varepsilon}(t)\right)-H_{e}\left(z_{\varepsilon}(s)\right)\right), \varphi-z_{\varepsilon}(s)\right) \\
= & A+B+C .
\end{aligned}
$$


Let us consider the following partition of $\Omega$ :

$$
\Omega=\left(z_{\varepsilon}(s) \geq \varepsilon\right) \cup\left(\left(z_{\varepsilon}(s) \leq \varepsilon\right) \cap\left(\varphi>z_{\varepsilon}(s)\right)\right) \cup\left(\varphi \leq z_{\varepsilon}(s)<\varepsilon\right)
$$

where on $\left(z_{e}(s)>\varepsilon\right)$

$$
\begin{array}{r}
H_{\varepsilon}\left(z_{\varepsilon}(s)\right)-1=0 \\
H_{\varepsilon}\left(z_{\varepsilon}(t)\right)-H_{\varepsilon}\left(z_{\varepsilon}(s)\right)=0
\end{array}
$$

and on $\left(z_{\varepsilon}(s) \leq \varepsilon\right) \cap\left(\varphi>z_{\varepsilon}(s)\right)$

$$
\begin{array}{r}
\left(H_{e}\left(z_{\varepsilon}(s)\right)-1\right)\left(\varphi-z_{\varepsilon}(s)\right)(e(s)-e(t)) \leq 0 \\
\left.e(t)\left(H_{\varepsilon}\left(z_{\varepsilon}\right)\right)-H_{\varepsilon}(z(s))\right)\left(\varphi-z_{\varepsilon}(s)\right) \geq 0 .
\end{array}
$$

So

$$
\begin{aligned}
& B \leq \int_{\left(\varphi \leq z_{\varepsilon}(s)<e\right)}\left(H_{\varepsilon}\left(z_{\varepsilon}(s)\right)-1\right)(e(s)-e(t))\left(\varphi-z_{e}(s)\right) \leq \int_{\Omega} 4 \varepsilon(e(s)-e(t)) \\
& C \leq \int_{\left(\varphi \leq z_{\varepsilon}(s)<e\right)} e(t)\left|H_{\varepsilon}\left(z_{\varepsilon}(t)\right)-H_{\varepsilon}\left(z_{\varepsilon}(s)\right)\right|\left|\varphi-z_{\varepsilon}(s)\right| \leq \int_{\Omega} 4 \varepsilon e(t)
\end{aligned}
$$

and (6.4) can be rewritten now as

$$
a\left(z_{\varepsilon}(t)-z_{\varepsilon}(s), \varphi-z_{\varepsilon}(s)\right) \leq A+\int_{\Omega} 4 \varepsilon(e(s)-e(t))+\int_{\Omega} 4 \varepsilon e(t) .
$$

Letting $\varepsilon \rightarrow 0$, we obtain (6.3). Dividing (6.3) by $t-s$ and letting $t \rightarrow s$ we get

$$
a\left(\frac{\partial^{+} z}{\partial t}, v-z(t)\right) \leq\left(\frac{\partial F}{\partial t}(t), v-z(t)\right)+\langle G, v-z(t)\rangle
$$

for all $v \in K$ and a.e. in $[0, T]$ as $\frac{\partial^{+} z}{\partial t}=\frac{\partial z}{\partial t}$ a.e. in $[0, T]$. Then $(6.1)$ is gained.

From (6.1) with $v=0$ and $v=2 z(t)$ we have

$$
a\left(\frac{\partial z}{\partial t}, z(t)\right)=\left(\frac{\partial F}{\partial t}, z(t)\right)+\left\langle\frac{\partial G}{\partial t}, z(t)\right\rangle
$$

and from problem. $\left(\mathrm{P}_{2}\right)$ we get, for all $\varphi \in K$,

$$
\begin{gathered}
a(z, \varphi) \geq(F, \varphi)+\langle G, \varphi\rangle \\
a(z, z)=(F, z)+\langle G, z\rangle .
\end{gathered}
$$

From (6.7), by time derivation, we have

$$
2 a\left(z, \frac{\partial z}{\partial t}\right)=\left(F, \frac{\partial z}{\partial t}\right)+\left(\frac{\partial F}{\partial t}, z\right)+\left\langle\frac{\partial \dot{G}}{\partial t}, z\right\rangle+\left\langle G, \frac{\partial z}{\partial t}\right\rangle .
$$

Then (6.5) and (6.8) give

$$
a\left(z, \frac{\partial z}{\partial t}\right)=\left(F, \frac{\partial z}{\partial t}\right)+\left\langle G, \frac{\partial z}{\partial t}\right\rangle
$$

and with (6.6) we gain (6.2). 
Theorem 6.2. Assume (H.0) - (H.3), $V=0, W \geq Q$ and $\left(\frac{\partial h}{\partial t}+h_{0}-h\right) \chi_{0} \leq 0$. Then if $\theta_{0}=\chi_{0}$, the solution of problem $\left(\mathrm{P}_{5}\right)$ is also solution of the problem $\left(\mathrm{P}_{0}^{\prime}\right)$ and $\theta=\chi$ a.e. in $\Omega \times(0, T)$.

Proof. From Theorem 5.1, if $(p, \theta)$ is the unique solution of problem $\left(\mathrm{P}_{5}\right)$, then $z$ defined by (3.8) is the unique solution of problem $\left(\mathrm{P}_{6}\right)$. Using the fact that $\theta_{0}=\chi_{0}$ and Theorem 6.1 , we find that $z$ is also the unique solution of problem $\left(\mathrm{P}_{1}{ }^{\prime}\right)$, and Theorem 3.4 end the proof.

\section{Conclusions and remarks}

As problem (PZ) has been deduced from a physical model using both $\theta$ and $p$ as unknowns while problem $\left(\mathrm{P}_{2}\right)$ has been deduced directly from another model of Hele-Shaw type when $\theta$ does not appears, the connection between them is precised; which enables us to establish Hele-Shaw problem as a particular case of lubrication in the last theorem.

In Theorems 5.1 and 5.4 we have neither a condition on $\frac{\partial h}{\partial t}$ nor on the regularity of the free boundary $\Gamma(t)$.

Theorem 7.1. Assuming (H.0) - (H.3), if $(p, \theta)$ is solution of problem $\left(\mathrm{P}_{4}\right)-\left(\mathrm{P}_{5}\right)$, then $z$ is the unique solution of the following variational problem:

$\left(\mathbf{P}_{1}^{\prime \prime}\right)$ Find $z(t) \in K$ for each $t \in(0, T)$ such that

$$
\begin{aligned}
a(z, \varphi-z) & \geq(f, \varphi-z)+\langle Q, \varphi-z\rangle \quad(\varphi \in K) \\
z(0) & =0
\end{aligned}
$$

with $f=\theta_{0} h_{0}-h$.

The proof of this statement is similar to that of Theorem 5.1, by choosing $\varphi=\psi-z$.

Theorem 7.2. If $z$ is the unique solution of the above variational inequality of first kind, then $z \in W^{1, \infty}\left(0, T ; H^{1}(\Omega)\right) \cap L^{\infty}\left(0, T ; H^{2}(\Omega) \cap \mathbb{V}\right), z \geq 0$ and $\frac{\partial z}{\partial t} \in$ $L^{\infty}\left(0, T ; H^{1}(\Omega) \cap K\right)$. Moreover, assume (H.0) - (H.3), $W \geq Q$ and $\left(\frac{\partial h}{\partial t}+h_{0}-h\right) \theta_{0} \leq 0$. Then we deduce that

$$
\left(12 \frac{\nu}{l^{3}} \frac{\partial z}{\partial t}, \theta\right) \quad \text { with } \theta= \begin{cases}1 & \text { in } \Omega(t) \\ \left(\theta_{0} h_{0}\right) / h & \text { in } \Omega^{0}(t)=\Omega \backslash \Omega(t)\end{cases}
$$

satisfies the problem $\left(\mathrm{P}_{3}\right)$ with $V=0$.

Proof. The proof is similar to that of Theorem 3.4. To prove that the $\theta$ so defined satisfies problem $\left(\mathrm{P}_{3}\right)$ with $V=0$, we choose $\varphi=\psi+z$ in (7.1) with $0 \leq \psi \in$ $H_{0}^{1}\left(\Omega^{0}(t)\right)$. 


\section{References}

[1] Agmon, S., Douglis, A. and L. Nirenberg: Estimates near the boundary for solutions of elliptic partial differential equations satisfying general boundary conditions. Part I. Comm. Pure Appl. Math. 12 (1959), 623 - 727.

[2] Allain, G.: Small-time existence for Navier-Stokes equations with a free surface. Appl. Math. Optim. 16 (1987), 37 - 50.

[3] Baiocchi, C.: Sur un probleme de frontiere libre traduisant le filtrage de ligides a travers des milieux poreux. Comptes Rendus Acad. Sci. Paris 273 A (1971), 29 - 32.

[4] Bayada, G. and M. Chambat: The Transition between the Stokes Equations and the Reynolds Equation: a Mathematical Proof. Appl. Anal. Optim. 14 (1986), 73 - 93.

[5] Begehr, H. and R. P. Gilbert: Hele-Shaw flows in $\mathbb{R}^{n}$. Nonlin. Anal. 10 (1986), 65 - 85.

[6] Begehr, H. and R. P. Gilbert: Non-Newtonian Hele-Shaw flows in $\mathbb{R}^{n}$. Nonlin. Anal. 11 (1987), 17 - 47.

[7] Bemelmans, J.: Liquid drops in a viscous fluid under the influence of gravity and surface tension. Manuskr. Math. 36 (1981), $105-123$.

[8] Bemelmans, J. and A. Friedman: Analyticity for the Navier-Stokes equations gouverned by surface tension on the free boundary. J. Diff. Equ. 55 (1984), 135 - 150.

[9] Benilan, P.: Equations de'évolution dans un espace de Banach quelconque et applications. Thesis. Orsay (France) 1972.

[10] Bayada, G., Boukrouche, M. and M. El Alaoui: Du probleme de Hele-Shaw au probleme de la cavitation en lubrification hayrodynamique. Preprint. Publication de l'equipe d'anlyse numerique Lyon-St. Etienne Nr. 100 (1990), 1 - 37.

[11] Breziz, H.: Problemes unilateraux. J. Math. Pures Appl. 51 (1972), 1 - 168.

[12] Brezis, H.: Analyse Fonctionelle Theorie et Aplications. Paris - New York - Barcelone: Masson 1983.

[13] Carrillo, j., Diaz, J. I. and S. J. Alavarez: Cavitation in lubrication: An evolution model. Congr. Mat. Apl. 25 - 29.09.1989 Fuengirola (Malaga).

[14] Capriz, G. and G. Cimati: Partial lubrication of full cylindrical bearings. Report. Cons. Naz. Ricerche Pisa 97-4 (1979), 1 - 23.

[15] Chaomleffel, J. P.: Influence des forces d'inertie en lubrification hydrodynamique. These Mecanique. Inst. Nat. Sci. Appl. Lyon 1983.

[16] Cizek, P. and V. Janovsky: Hele-Shaw flows model of the injection by a point source. Proc. Royal Soc. Edinburgh (Section A) 91 (1981), 147 - 159.

[17] Coppoletta, G.: Su un problema a frontiera libera di evoluzione connesso al moto di un fluido. Due Lastre Piane. Inst. Lombardo (Rend. Sci.) A 115 (1981), 361 - 379.

[18] Di Benedetto, E. and A, Friedman: The ill-posed Hele-Shaw model and the Stefan problem fo supercooled water. Trans. Amer. Math. Soc. 282 (1984):

[19] El Alaoui, M. T.: Sur u probleme a frontiere libre en mecanique des films minces. These Math. Univ. Lyon 1, Nr. 67 - 86 (1986).

[20] Elliott, C. M. and V. Janovsky: A variational inequality approach to the Hele-Shaw flows with a moving boundary. Proc. Royal Soc. Edinburgh (Section A) 88 (1981), 93 - 107.

[21] Dowson, D., Godet, M. and C. M. Taylor: Cavitation and Related Phenomeny in Lubrication. London: Mech. Eng. Publ. Ltd. 1975. 
[22] Gilardi, G.: A new approach to evolution free boundary problems. Comm. Part. Diff. Equ. 4 (1979), 1099 - 1122.

[23] Gilbert, R. P. and Wen Guo Chun: Free boundary problems occuring in planar fluid dynamics. Nonlin. Anal. 13 (1989), 285 - 303.

[24] Gilbert, R. P. and P. Shi: Anisotropic Hele-Shaw flows. Math. Methods in Appl. Sci. 11 (1989), 417 - 429.

[25] Meyer, G. H.: Hele-Shaw flows with A Cusping free boundary. J. Comp. Phys. 44 (1981), $262 \cdot 276$.

[26] Gustafsson, B.: Applications of variational inequalities to a moving boundary problem for Hele-Shaw flow. SIAM J. Math. Anal. 16 (1985), 279 - 300.

[27] Gustafsson, B.: On a differential equation arising in Hele-Shaw flow moving boundary problem (Trita-Mat.: Vol. 36). Math. Royal Inst. Tech. Stockholm 1981.

[28] Howison, S. D.: Cups development in Hele-Shaw flow, with a free surface. SIAM J. Appl. Math. 46 (1986), $20-26$.

[29] Richardson, S.: Hele-Shaw flows with a free boundary produced by the injection of fluid into a narrow channel. J. Fluid Mech. 56 (1972), $609-618$.

[30] Richardson, S.: Some Hele-Shaw flows with time-dependent free bondaries. J. Fluid Mech. 102 (1981), 263 - 278.

[31] Rodrigues, J. F.: Obstacle Problems in Mathematical Physics (Math. Studies: Vol. 134). Amsterdam: North-Holland Publ. Comp. 1987.

[32] Sakai, M.: Quadrature Domains (Lect. Notes Mat.: Vol. 934). Berlin: Springer - Verlag 1982.

[33] Sakai, M.: Applications of variational inequalities to the existence theorem on quadrature domains. Trans. Amer. Math. Soc. 276 (1983), 267 - 279.

[34] Beale, J. Thomas: The initial value problem for the Navier-Stokes equations with a free surface. Comm. Pure Appl. Math. 34 (1981), 359 - 392.

[35] Vinogradov, Yu. P. and P. P. Kufarev: On a seepage problem (in Russian). Prikl. Math. Mekh. 12 (1948).

[36] Wehausen, J. V. and E. V. Laitone: Surface waves. In: Handbuch der Physik: Vol. 9. Berlin: Springer - Verlag 1960, pp. $446 \cdot 778$. 